\title{
Zeolitic Imidazolate Framework-Mediated Synthesis of Co304 Nanoparticles Encapsulated in N-doped Graphitic Carbon as an Efficient Catalyst for Selective Oxidation of Hydrocarbons
}

Saikiran Pendem, Ramana Singuru, Chitra Sarkar, Boby Joseph, Jyh-Fu Lee, Digambar Balaji Shinde, Zhiping Lai, and John Mondal

ACS Appl. Nano Mater., Just Accepted Manuscript• DOI: 10.1021/acsanm.8b01027 • Publication Date (Web): 28 Aug 2018

Downloaded from http://pubs.acs.org on September 2, 2018

\section{Just Accepted}

"Just Accepted" manuscripts have been peer-reviewed and accepted for publication. They are posted online prior to technical editing, formatting for publication and author proofing. The American Chemical Society provides "Just Accepted" as a service to the research community to expedite the dissemination of scientific material as soon as possible after acceptance. "Just Accepted" manuscripts appear in full in PDF format accompanied by an HTML abstract. "Just Accepted" manuscripts have been fully peer reviewed, but should not be considered the official version of record. They are citable by the Digital Object Identifier (DOI®). "Just Accepted" is an optional service offered to authors. Therefore, the "Just Accepted" Web site may not include all articles that will be published in the journal. After a manuscript is technically edited and formatted, it will be removed from the "Just Accepted" Web site and published as an ASAP article. Note that technical editing may introduce minor changes to the manuscript text and/or graphics which could affect content, and all legal disclaimers and ethical guidelines that apply to the journal pertain. ACS cannot be held responsible for errors or consequences arising from the use of information contained in these "Just Accepted" manuscripts. 


\title{
Zeolitic Imidazolate Framework-Mediated Synthesis of $\mathrm{Co}_{3} \mathrm{O}_{4}$ Nanoparticles Encapsulated in N-doped Graphitic Carbon as an Efficient Catalyst for Selective Oxidation of Hydrocarbons
}

\author{
Saikiran Pendem, ${ }^{\dagger}, \#$ Ramana Singuru, ${ }^{\dagger, \#}$ Chitra Sarkar, ${ }^{\dagger, \#}$ Boby Joseph, ${ }^{\ddagger}$ Jyh-Fu Lee, ${ }^{\S}$ \\ Digambar B. Shinde," Zhiping Lai" and John Mondal*,ं,\# \\ †Inorganic \& Physical Chemistry Division, CSIR-Indian Institute of Chemical Technology, \\ Uppal Road, Hyderabad-500007, India. Email: johncuchem@gmail.com; \\ johnmondal@iict.res.in \\ ${ }^{\ddagger}$ Elettra-Sincrotrone Trieste, S.S. 14, Km 163.5 in Area Science Park, Basovizza 34149, \\ Trieste, Italy \\ ${ }^{\S}$ National Synchrotron Radiation Research Center, 101 Hsin-Ann Road, Hsinchu 30076, \\ Taiwan. \\ 'Division of Physical Science and Engineering, King Abdullah University of Science and \\ Technology (KAUST), Thuwal 23955-6900, Saudi Arabia. \\ \#AcSIR - Indian Institute of Chemical Technology, Hyderabad-500007, India.
}

\begin{abstract}
:
The aerobic solvent-free selective oxidation of the $\mathrm{C}-\mathrm{H}$ bonds of hydrocarbons to the corresponding ketones by earth-abundant catalysts would provide high added value both from economy and environment points of view. In this respect herein, $\mathrm{Co}_{3} \mathrm{O}_{4}$ nanocrystals embedded into $\mathrm{N}$-doped graphitic carbon nanohybrids $\left(\mathrm{Co}_{3} \mathrm{O}_{4} @\right.$ GNC) are prepared by uniform seed-mediated growth and deposition of Co-based zeolitic imidazolate framework-9 (ZIF-9) nanocrystals on graphene oxide (GO) nanosheets followed by facile carbonization of the ZIF-GO composite under inert atmosphere at high temperature. The specific textural and chemical characteristics of as-synthesized nanohybrids at different pyrolysis temperatures were comprehensively investigated by performing various spectroscopic tools. N-doped graphitic carbon wrapped $\mathrm{Co}_{3} \mathrm{O}_{4}$ nanocrystals is demonstrated to be an efficient oxidation catalyst of arylalkanes. Under the optimized reaction conditions, $\mathrm{Co}_{3} \mathrm{O}_{4} @$ GNC-B catalyst (as-synthesized at $700^{\circ} \mathrm{C}$ carbonization temperature) was found to exhibit superior catalytic performance with improved stability (reproducible conversion values upon sixth cycles) in ethyl benzene oxidation, providing $65.8 \%$ conversion of ethyl benzene with an exclusive selectivity of $72.6 \%$ for acetophenone. With the help of combined catalytic studies and the different characterization methods including XPS and XAFS analysis we can emphasis that the presence of Co- $\mathrm{N}_{x}$ active site and the synergistic effect between $\mathrm{Co}_{3} \mathrm{O}_{4}$ nanocrystals and unique $\mathrm{N}$-containing interconnected carbonaceous framework is responsible for the superior
\end{abstract}


catalytic activity and stability of $\mathrm{Co}_{3} \mathrm{O}_{4} @$ GNC. Moreover, the $\mathrm{Co}_{3} \mathrm{O}_{4} @$ GNC catalyst could also be explored for a variety of arylalkane substrates in oxidation reaction with high catalytic activity. Furthermore future studies could be extended on the design of precious-metal-free robust catalysts as-derived from ZIFs for advanced applications in fine-chemical production.

KEYWORDS: $\mathrm{N}$-doped carbon $\cdot \mathrm{Co}_{3} \mathrm{O}_{4} \cdot$ Zeolitic Imidazolate Frameworks $\bullet$ Solvent-free oxidations $\bullet$ Hydrocarbons

\section{INTRODUCTION:}

Fine chemicals productions with the selective oxidation of saturated or unsaturated hydrocarbons has received a staggering degree of attention both in view of economy and environment. ${ }^{1-3}$ During the past few decades, extensive efforts have focused in designing and developing of most promising and efficient catalysts for selective transformation of organic hydrocarbons and alcohols. To site some examples, Wang and co-workers have developed $\mathrm{Pd} @ \mathrm{~N}-$ doped carbon from glucose which exhibited solvent-free aerobic oxidation of hydrocarbons. ${ }^{4}$ Dai and coworkers have successfully designed Pd clusters encapsulated in hybrid core-shell and found their excellent catalytic performance in oxidation of hydrocarbons and alcohols. ${ }^{5} \mathrm{Li}$ and co-workers have studied selective solvent-free aerobic oxidation of saturated hydrocarbons using Au-Pd alloy dispersed on a zeolite-type metalorganic framework (i.e., MIL-101). ${ }^{6}$ Although superior catalytic activity could be achieved with these noble metal based catalysts but the poor stability, higher cost and limited abundance in the earth's crust are big hurdles for their extensive applications in industry. A literature survey has brought into focus the need towards exploring robust non-noble metal catalysts in replacement of expensive ones for carrying out oxidation of aromatic hydrocarbons. Below we cite some examples of research in this direction. Liu et al. have designed efficient hydrocarbon oxidation catalysts $\mathrm{Co} @ \mathrm{GCNs}$ by encapsulation of cobalt nanoparticles into graphitic nitrogen-doped carbon nanotube hybrids. ${ }^{7}$ Noble-metal-free Co$\mathrm{N}-\mathrm{C}$ immobilized carbon nanotubes (CNTs) as prepared via thermal treatment of nitrogenrich cobalt tetraphenyl porphyrin (CoTPP) supported on CNTs have been utilized as a potential ethylbenzene oxidation catalyst by Liu and co-workers. ${ }^{8}$ Similarly, this research group have designed nitrogen-doped carbon cobalt (Co-N-C) grafted on graphitic carbon nitride $\left(\mathrm{g}-\mathrm{C}_{3} \mathrm{~N}_{4}\right)$ catalysts $\left(\mathrm{Co}-\mathrm{N}-\mathrm{C} / \mathrm{g}-\mathrm{C}_{3} \mathrm{~N}_{4}\right)$ which exhibited enhanced catalytic performance for ethylbenzene oxidation. ${ }^{9}$ Iron nanowire filled carbon nanotubes (Fe@CNTs) has been reported with superior catalytic activity for selective oxidation of ethylbenzene by Peng et $a l .{ }^{10}$ In contrast, carbon nanotubes (CNTs) and carbon nanotubes with doped nitrogen atoms 
in graphitic domains (NCNTs) as metal-free catalysts for hydrocarbon oxidation have been designed by Peng and co-workers but its practical application yet remains unexplored owing to the use of additives and oxidants including toxic metal oxides and peroxides to improve the catalytic performance and selectivity of the desired products. ${ }^{11,12,13}$

Zeolitic imidazolate framework (ZIFs) is a subclass of metal organic frameworks (MOFs), a type of crystalline inorganic-organic hybrid materials that have attracted extensive awareness in various applications owing to their inherent properties including high thermal and chemical stability, large pore volume with tuneable pore size distribution and high specific surface area. ${ }^{14,15}$ ZIFs can serve as precursors to synthesize networked porous carbon materials especially with heteroatoms (such as nitrogen) doping, upon heat treatment in inert atmospheres which become a new and versatile technique without using external templates. The dual role of the nitrogen containing ZIFs could be attributed of being a precursor for carbon and as a source of nitrogen. A great deal of research has been conducted to develop successfully hierarchically nanoporous metal-containing carbonaceous materials employing MOFs as precursors by direct carbonization technique under inert atmosphere. ${ }^{16,17}$ These heteroatom doped metal or metal oxide containing nanoporous carbon materials as derived from MOF precursors have been regarded as promising candidates in catalysis, ${ }^{18,19}$ sensing, ${ }^{20}$ batteries, ${ }^{21}$ adsorption, ${ }^{22}$ and energy storage, ${ }^{23}$ due to their chemical resistance, excellent electrical conductivity and high porosity enough to expose more active sites and enhance the mass transport. To date, there are only few recent reports focusing on the design and synthesis of metal or metal oxide encapsulated hierarchically 3D porous carbons via direct carbonization of MOF precursors employed as catalysts. Lin et al. have developed magnetic cobalt-graphene (MCG) nanocomposite by carbonizing ZIF-67 and graphene oxide (GO) which served as an excellent catalyst to activate peroxymonosulfate (PMS) in the advanced oxidation process. ${ }^{24}$ Zhao et al. developed graphene oxide/core-shell structured metal-organic framework nano-sandwiches derived cobalt/N-doped carbon nanosheets, which showed high oxygen reduction reactions. ${ }^{25}$ High performance oxygen reduction hierarchically porous Co$\mathrm{N}_{x} / \mathrm{C}$ catalysts have been designed by Wang et al. by carbonization of graphene oxide (GO) supported ZIF nanocrystal arrays followed by acid leaching process. ${ }^{26}$ Ma and co-workers demonstrated a facile synthesis strategy of $\mathrm{Co} @ \mathrm{Co}_{3} \mathrm{O}_{4}$ encapsulated $\mathrm{N}$-doped mesoporous carbon cages on reduced graphene oxide (r-GO) as an active oxygen-evolving catalyst. ${ }^{27} \mathrm{~A}$ special attention has been directed towards graphene oxide with a unique two-dimensional structure and excellent electrical conductivity as a promising candidate of catalytic support. 
We employ the self assembly strategy of ZIF-9 and graphene oxide (GO) via electrostatic attraction to form a carbon-based composite material, which upon carbonization route at high temperature under inert atmosphere was adopted to encapsulate novel $\mathrm{Co}_{3} \mathrm{O}_{4}$ nanoparticles into nitrogen-doped graphitic carbon. Herein, we further demonstrate that graphene oxide wrapped ZIF-9 microcrystals could be considered as useful precursors for the preparation of $\mathrm{N}$-doped interconnected 3D-porous network. All the developed nanocatalysts have been abbreviated as $\mathrm{Co}_{3} \mathrm{O}_{4} @$ GNC in the present manuscript. The specific textural, chemical characteristics and atomic-level relationship between structure and catalysis have been throughly investigated by performing powder $\mathrm{XRD}, \mathrm{N}_{2}$ physisorption, high-resolution transmission electron microscopy (HR-TEM), high angle annular dark field scanning transmission electron microscopy (HAADF-STEM), energy-dispersive X-ray (EDX) analysis, scanning electron microscopy (SEM) with the corresponding elemental mapping, Co K-edge X-ray absorption fine structure (XAFS), Raman and X-ray photoelectron spectroscopy (XPS) studies. The $\mathrm{Co}_{3} \mathrm{O}_{4} @$ GNC nanohybrid exhibited an impressive catalytic performance in the controlled oxidation of saturated and unsaturated hydrocarbons under aerobic and solvent-free conditions. In terms of catalyst stability, these designed catalysts afford much higher activity with good recyclability (upto $6^{\text {th }}$ catalytic run) for oxidation of diverse alkanols relative to the conventional reported catalysts under the same reaction conditions to demonstrate the benefit of such carbonaceous nanoarchitecture in heterogeneous nanocatalysis. On the basis of the detailed investigation we could speculate that the advanced catalytic performance and durability of $\mathrm{Co}_{3} \mathrm{O}_{4} @ \mathrm{GNC}$ catalysts is connected with the synergistic effect generated in between Co-N-C and carbonaceous materials, as experimentaly evidenced with XPS and XAFS tools. We expect that these results will encourage further extended research in exploring novel non-noble metal based efficient catalyst systems for the application of ambient air and recyclable cobalt catalysts in finechemical production with high activity.

\section{EXPERIMENTAL SECTION:}

\section{Catalyst preparation:}

\section{Synthesis of $\mathrm{Co}_{3} \mathrm{O}_{4} @$ GNC-A, B and $\mathrm{C}$ :}

In a typical synthesis procedure, $\mathrm{Co}\left(\mathrm{NO}_{3}\right)_{2} .6 \mathrm{H}_{2} \mathrm{O}(1.44 \mathrm{mmol}, 0.420 \mathrm{~g})$ and benzimidazole $(2.87 \mathrm{mmol}, 0.340 \mathrm{~g})$ were mixed together in $80 \mathrm{~mL} \mathrm{~N}$,N-dimethylformamide to get a clear pink colour solution and it was allowed to stir at room temperature for $1 \mathrm{~h}$. GO was first prepared from natural graphite using a modified method as reported elsewhere. ${ }^{28}$ Then 0.190 $\mathrm{g}$ graphene oxide was added to the solution and sonicated for $2 \mathrm{~h}$ to ensure homogeneity with 
well-dispersed graphene oxide solution. The resulting mixture was stirred for $2 \mathrm{~h}$ at room temperature and then solvothermally treated at $130^{\circ} \mathrm{C}$ for $48 \mathrm{~h}$. After cooling down to room temperature, the as-synthesized blue colour ZIF-graphene oxide composites were collected by centrifugation and washed several times with methanol, and then dried at room temperature. The obtained blue colour ZIF-GO solid was finely ground into powder and transferred to a quartz boat, heated in a tubular furnace at 600,700 and $800^{\circ} \mathrm{C}$ temperatures, respectively, with the heating rate of $5^{\circ} \mathrm{C} \mathrm{min}^{-1}$ under $\mathrm{N}_{2}$ atmosphere for $3 \mathrm{~h}$ and cooled down to room temperature naturally to yield black color solid $\mathrm{Co}_{3} \mathrm{O}_{4} @$ GNC-A, $\mathrm{Co}_{3} \mathrm{O}_{4} @$ GNC-B and $\mathrm{Co}_{3} \mathrm{O}_{4} @ \mathrm{GNC}-\mathrm{C}$, respectively.

\section{Aerobic oxidation of arylalkanes under solvent-free condition catalyzed by $\mathrm{Co}_{3} \mathrm{O}_{4} @$ GNC:}

Aerobic oxidation of arylalkanes under solvent-free conditions catalyzed by $\mathrm{Co}_{3} \mathrm{O}_{4} @ \mathrm{GNC}$ catalysts were carried out under batch reaction conditions. In a typical catalytic oxidation of arylalkane, a $50 \mathrm{ml}$ stainless-steel reactor inbuilt with a pressure gauge setup lined with Teflon was charged with a mixture of ethylbenzene $(25 \mathrm{~mL})$ and $50 \mathrm{mg}$ of $\mathrm{Co}_{3} \mathrm{O}_{4} @$ GNC catalyst. Then the reactor was fitted with an overhead stirrer, pressurized with air to the desired pressure of 20 bar with continuous purging by air. The temperature of the autoclave was fixed at $140^{\circ} \mathrm{C}$ for the desired reaction time with continuous stirring at a speed of 800 rpm. After the reaction finish, autoclave pressure has been slowly released and catalyst was recovered from the reaction mixture by centrifugation for $\mathrm{GC}$ analysis of the reaction mixture. Gas chromatograph (Shimadzu 2010) equipped with a flame ionization detector with no wax capillary column (diameter: $0.25 \mathrm{~mm}$, length: $30 \mathrm{~m}$ ) and GC-MS (Shimadzu, GCMSQP2010S) have been utilized for quantitative analysis and product identification in the presence of 1,4-dichlorobenzene as internal standard.

\section{Recycling tests:}

In a typical recycling experiment of aerobic oxidation of arylalkanes under solvent-free conditions with $\mathrm{Co}_{3} \mathrm{O}_{4} @ \mathrm{GNC}$, a mixture of ethyl benzene $(75 \mathrm{~mL})$ and catalyst $(150 \mathrm{mg})$, was heated with stirring at $140^{\circ} \mathrm{C}$ in a $200 \mathrm{~mL}$ dry stainless-steel reactor pressurized with air at 20 bar for $4 \mathrm{~h}$. Then the blackish solid was recovered from the reaction mixture by centrifugation, and used for next catalytic run in succession after proper washing with methanol and drying at $80^{\circ} \mathrm{C}$ for $12 \mathrm{~h}$.

\section{Hot filtration test:}

We have performed hot filtration test to authenticate heterogeneous and robust nature of our newly designed $\mathrm{Co}_{3} \mathrm{O}_{4} @$ GNC catalyst and no substantial leaching of the cobalt metals into 
the solution took place. Typically, a $50 \mathrm{~mL}$ dry stainless-steel reactor was filled with a mixture of ethyl benzene $(25 \mathrm{~mL})$ and catalyst $(50 \mathrm{mg})$ under 20 bar air pressure and heated at $140^{\circ} \mathrm{C}$ for $2 \mathrm{~h}$. After $2 \mathrm{~h}$, the catalyst was isolated from the hot reaction mixture by centrifugation and we have achieved 52.8\% ethyl benzene conversion over $\mathrm{Co}_{3} \mathrm{O}_{4} @$ GNC-B catalyst (by GC analysis). Then, we have conducted the oxidation reaction with the filtrate with the same reaction mixture in a stainless-steel reactor under identical conditions for a further $6 \mathrm{~h}$ to ensure whether any leaching of cobalt species occurred. After $6 \mathrm{~h}$, no obvious enhancement in the ethyl benzene conversion beyond 52\% was accomplished as determined by GC analysis.

\section{RESULTS AND DISCUSSION:}

The overall synthetic strategy for the preparation of $\mathrm{Co}_{3} \mathrm{O}_{4}$ nanoparticles (NPs) encapsulated on graphitic N-doped carbon is illustrated in Scheme 1. Herein, we have adopted ZIF seedmediated deposition route to prepare ZIF-GO nanocomposite, where the uniform deposition of Co-ZIF nanocrystals on GO nanosheets took place. Firstly, previously reported modified method has been employed to synthesize graphene oxide (GO) with epoxy and hydroxyl functional groups. ${ }^{28}$ Secondly, Co based ZIF has been prepared considering benzimidazole as the ligand and $\mathrm{Co}\left(\mathrm{NO}_{3}\right)_{2} \cdot 6 \mathrm{H}_{2} \mathrm{O}$ as the metal source and subsequently aqueous $\mathrm{GO}$ solution was introduced after the formation of the Co-ZIF. ${ }^{29}$ For instance, easy preparation, controllable crystalline size, good thermal stability and high contents of carbon and nitrogen of ZIF-9 are beneficial for making it an ideal cobalt-based ZIF precursor for the synthesis of Co-based catalysts with abundant active catalytic sites. The characteristic peaks appeared in the wide angle powder XRD pattern of Co-ZIF (Fig. S1, SI), is in good agreement with the previous literature report. ${ }^{29}$ The nitrogen adsorption/desorption isotherms of the ZIF-9 (Fig. $\mathrm{S} 2$, SI) could be recognized as typical type I isotherms with a rapid $\mathrm{N}_{2}$ uptake at the relative lower $\mathrm{P} / \mathrm{P}_{0}$ pressure region. The strong attachment of the Co-ZIF crystal onto the GO layer could be favoured due to the coordination interactions between the $\mathrm{Co}^{2+}$ metal ions from the Co-ZIF and oxygen containing epoxy and hydroxyl groups from the GO sheet. Through the washing procedure with deionized water was followed to remove the loosely attached Co-ZIF nanocrystals from ZIF arrays supported on GO sheets (ZIF-GO). The FE-SEM images of ZIF-GO demonstrates that Co-ZIF nanocrystals having size around $80 \mathrm{~nm}$ are homogeneously deposited on the GO sheets to form a sandwich-like structure (Fig.S3, SI). The continuous arrangement of aggregated ZIF-9 nanocrystals wrapped by GO layers is observed from the high resolution TEM images (Fig.S4, SI), indicating a successful synthesis of ZIF-9 MOF supported on GO nanosheets without destroying polyhedron morphology of 
ZIF-9. ${ }^{25}$ Finally, the as-prepared ZIF-GO nanohybrid has been pyrolyzed in an inert atmosphere for $3 \mathrm{~h}$ at high temperatures $\left(600^{\circ} \mathrm{C}, 700^{\circ} \mathrm{C}\right.$ and $800^{\circ} \mathrm{C}$, respectively) to obtain $\mathrm{Co}_{3} \mathrm{O}_{4}$-NP confined with nitrogen doped carbon composites which are represented as $\mathrm{Co}_{3} \mathrm{O}_{4} @$ GNC-A, $\mathrm{Co}_{3} \mathrm{O}_{4} @$ GNC-B and $\mathrm{Co}_{3} \mathrm{O}_{4} @$ GNC-C, respectively. During the carbonization process at high temperature, the organic ligands from the ZIF are decomposed and condensed to form nitrogen doped carbonaceous skeleton, while the central $\mathrm{Co}^{2+}$ ions were transformed into $\mathrm{Co}_{3} \mathrm{O}_{4}$-NPs, which were in situ confined into nitrogen-carbon composite, leading to homogeneous dispersion of the metal NPs throughout carbon matrix with less aggregation and generation of highly catalytically active $\mathrm{Co}_{0} \mathrm{~N}_{x}$ sites with an effective approach for catalytic graphitization of amorphous carbon via solid-state transformation process. Additionally, homogeneous dispersion of the $\mathrm{Co}_{3} \mathrm{O}_{4}-\mathrm{NPs}$ and in situ generated nitrogen-doped carbonaceous architecture is highly favoured for the periodic arrangement of cobalt centres and organic ligands in ZIF scaffolds. In this route, the role of GO could be emphasized as a structure-directing agent to assemble ZIF nanocrystals on GO nanosheets. During the course of pyrolysis process under high temperature GO is transformed into reduced graphene oxide ( $\mathrm{rGO}$ ) which acts as a binder to unite individual ZIF-derived carbon nanoparticles into the porous structure, resulting an enhancement in overall conductivity. ${ }^{30}$ The cobalt contents in $\mathrm{Co}_{3} \mathrm{O}_{4} @$ GNC-A, $\mathrm{Co}_{3} \mathrm{O}_{4} @$ GNC-B and $\mathrm{Co}_{3} \mathrm{O}_{4} @$ GNC-C nanohybrid materials are $13.4,3.5$ and $7.7 \mathrm{mmolg}^{-1}$, respectively, as measured by inductively coupled plasma optical emission spectrometer (ICP-OES) analysis. Elemental $(\mathrm{C}, \mathrm{H}, \mathrm{N})$ analysis demonstrated that $\mathrm{N}$ has been successfully incorporated into the system (Table $\mathrm{S} 1$, $\mathrm{SI}$ ) and decrease in the $\mathrm{N}$ content (wt \%) with the increase of carbonization temperature could be explained by the partial destroy of $\mathrm{C}-\mathrm{N}$ bonds during the graphitization process. Furthermore, no significant change in cobalt content was found with the enhancement of carbonization temperature is in good agreement with the previous report by Liu and coworkers. ${ }^{7}$ The thermal stability of the as-synthesized ZIF-GO composite has been evaluated 


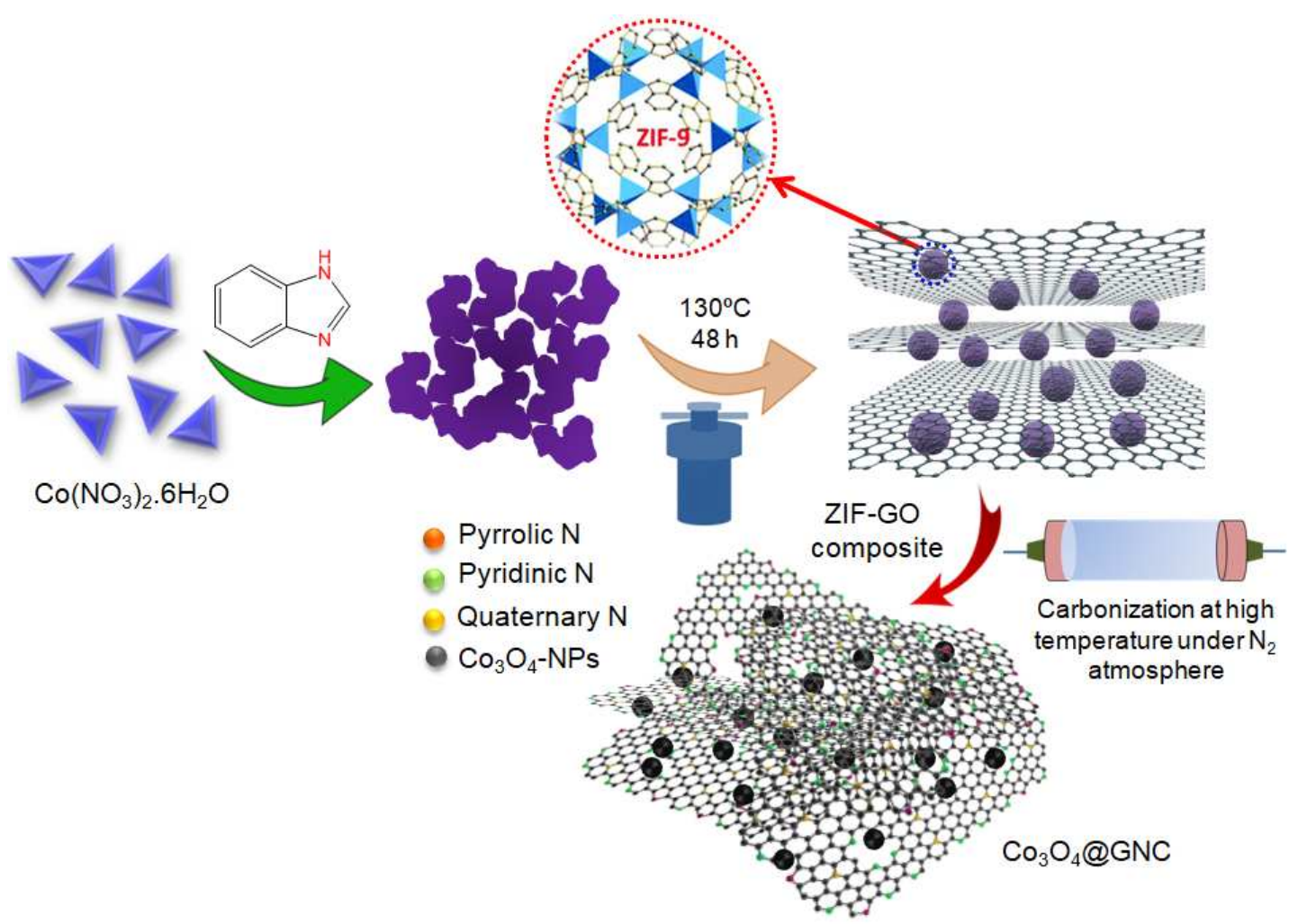

Scheme 1: Schematic illustration for metal organic framework-mediated synthesis of $\mathrm{Co}_{3} \mathrm{O}_{4}$ nanoparticles encapsulated in $\mathrm{N}$-doped graphitic carbon $\left(\mathrm{Co}_{3} \mathrm{O}_{4} @ \mathrm{GNC}\right)$

by thermogravimetric analysis (TGA). According to the TGA-DSC curves (Fig.S5, SI), it is understood that the ZIF-GO composite was highly stable up to $350^{\circ} \mathrm{C}$. The initial weight loss $\sim 3.8 \mathrm{wt} \%$ could be attributed to the removal of adsorbed water or trapped gas molecule in the porous framework of the ZIF. Along with the initial weight loss, a gradual weight loss $\sim 6.68$ $\mathrm{wt} \%$ has been detected at the temperature range of $200^{\circ} \mathrm{C}$ and become stable after $300^{\circ} \mathrm{C}$, which corresponds to the release of free hydroxyl groups, burning of the organic linkers followed by evaporation as small molecules.

The wide angle powder X-ray diffraction spectra of all the carbonized materials have been presented in the Fig.1a. The characteristic Bragg peaks appeared at $2 \theta$ values of $31.2,36.8$, $44.8,59.4$ and $65.2^{\circ}$, respectively corresponding to the (220), (311), (400), (511) and (440) crystalline reflections of spinel structured $\mathrm{Co}_{3} \mathrm{O}_{4}$ nanoparticles (JCPDS no. 42-1467). Diffraction pattern does not evidence any Bragg peaks corresponds to $\mathrm{Co}^{0}-\mathrm{NP} .^{31,32}$ Furthermore, in the XRD patterns of the nanohybrids it is difficult to distinguish diffraction peak at $2 \theta=26^{\circ}$ responsible for the (002) plane of graphitic carbon, which could be due to the disordered nature of the highly random stacking of the graphene nanosheets during the carbonation and annealing ${ }^{33,34}$ as well as the reduced structure factor for this particular Bragg 
reflection compared to that of the $\mathrm{Co}_{3} \mathrm{O}_{4}$-GNC. It is noticed that with the increase of the carbonization temperature the reflections of the $\mathrm{Co}_{3} \mathrm{O}_{4}$-GNC become more intense with narrower peak width, demonstrating an improved crystallization and particle size growth at elevated temperature. The average $\mathrm{Co}_{3} \mathrm{O}_{4}$ particle sizes estimated from the Scherrer equation considering $36.8^{\circ}$ (311) reflections are $7.85,4.83$, and $14.5 \mathrm{~nm}$ for samples obtained respectively at $600^{\circ} \mathrm{C}, 700^{\circ} \mathrm{C}$ and $800^{\circ} \mathrm{C}$. The above estimated particle sizes were in reasonable accordance with observed TEM results. ${ }^{35}$ It could be observed from the wide angle powder XRD patterns (Fig. 1a) the characteristic weak Bragg diffraction appeared at the $2 \theta$ of $42.5^{\circ}$ in between of $36.8^{\circ}$ and $44.8^{\circ}$ for $\mathrm{Co}_{3} \mathrm{O}_{4} @$ GNC-B \& $\mathrm{Co}_{3} \mathrm{O}_{4} @$ GNC-C, respectively, could be attributed to the graphitic carbon, which is similar to the commercial CNTs. ${ }^{8}$ The absence of this graphitic carbon peak in the PXRD pattern of the $\mathrm{Co}_{3} \mathrm{O}_{4} @$ GNCA could be ascribed to the fact that higher pyrolysis temperature is solely responsible to generate this type of nanostructure which is also experimentally evidenced from the TEM images.
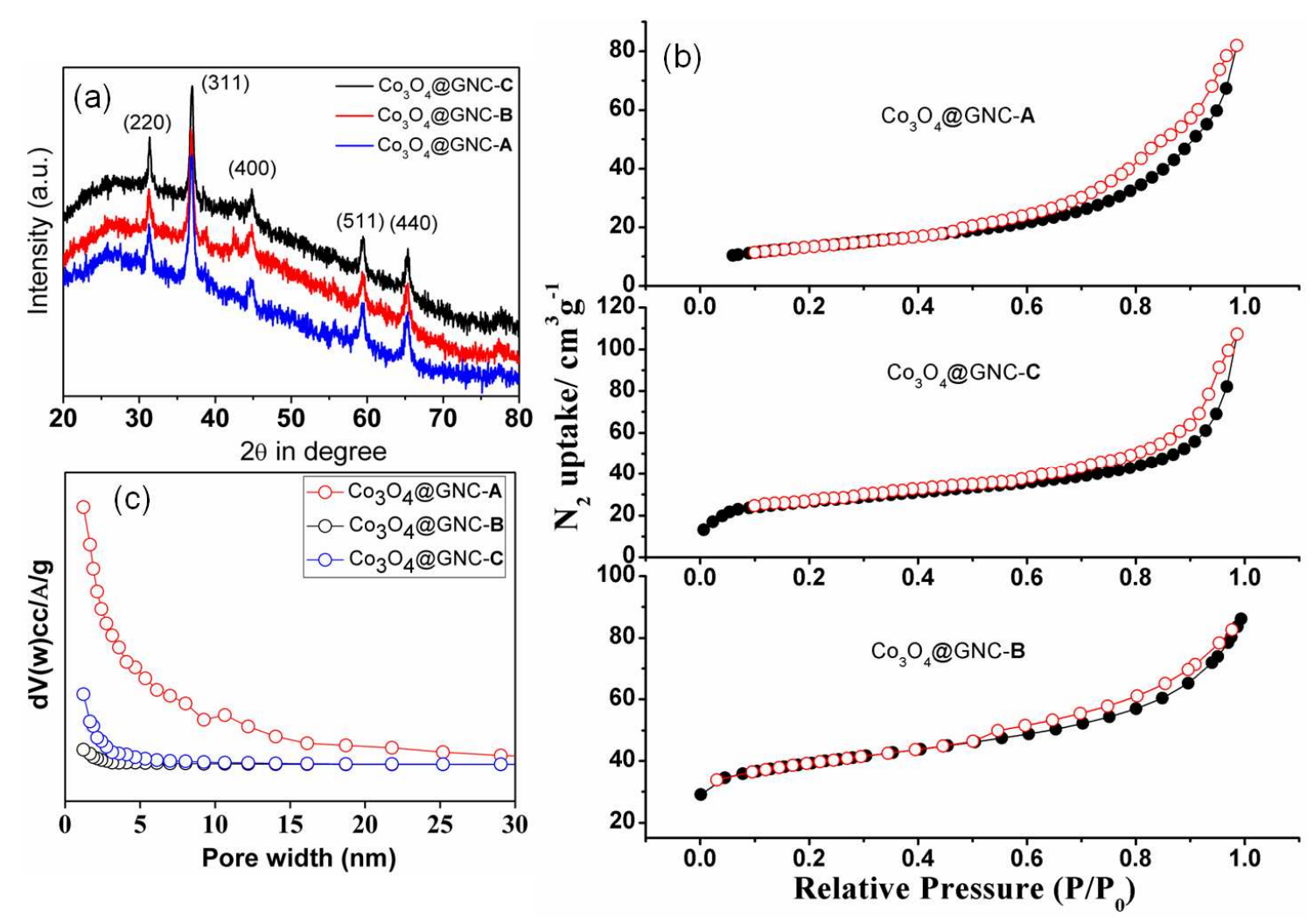

Figure 1: Wide angle power XRD patterns (a), $\mathrm{N}_{2}$ adsorption/desorption isotherms (b) and (c) with the corresponding pore size distributions of the respective $\mathrm{Co}_{3} \mathrm{O}_{4} @$ GNC-A, $\mathrm{Co}_{3} \mathrm{O}_{4} @$ GNC-B, and $\mathrm{Co}_{3} \mathrm{O}_{4} @$ GNC-C nanohybrids. 
The composition and the degree of graphitization of the $\mathrm{Co}_{3} \mathrm{O}_{4} @$ GNC nanohybrids have been evaluated by Raman spectroscopy (Fig.S6a). In the Raman spectra, two broad characteristic peaks, so called D band and the graphitic G band, located respectively at 1332 and $1585 \mathrm{~cm}^{-1}$, are observed whose relative intensity is closely related to the amount of disorder caused by lattice defects in the carbon materials. In particular, the $I_{\mathrm{D}} / I_{\mathrm{G}}$ ratio, the intensity ratio of these peaks can be correlated with the degree of graphitization. ${ }^{32}$ The $I_{\mathrm{D}} / I_{\mathrm{G}}$ ratio of $\mathrm{Co}_{3} \mathrm{O}_{4} @$ GNC materials decreased when the carbonization temperature was enhanced $\left(1.197,1.162\right.$ to 1.107 respectively for 600,700 and $\left.800^{\circ} \mathrm{C}\right)$ thus evidencing a higher degree of graphitization with increasing temperature. In addition, it has been noticed that the nitrogen content in the as-synthesized materials after carbonization has been retained at the low pyrolysis temperature (Table $\mathrm{S} 1, \mathrm{SI}$ ). The lower $I_{\mathrm{D}} / I_{\mathrm{G}}$ ratio of the $\mathrm{Co}_{3} \mathrm{O}_{4} @ \mathrm{GNC}$ nanohybrid compared with the GO $\left(I_{\mathrm{D}} / I_{\mathrm{G}}=1.3\right)$ illustrated that the delocalized $\pi$ conjugation was partially restored during the thermal carbonization of the ZIF-GO composite. ${ }^{35}$ The characteristic porous nature of $\mathrm{Co}_{3} \mathrm{O}_{4} @$ GNC nanohybrids were examined by $\mathrm{N}_{2}$ adsorption/desorption analysis measured at $77 \mathrm{~K}$ (Fig.1b), exhibited a typical type II isotherm, according to the IUPAC nomenclature. In all cases, the adsorption profiles display steady rise in nitrogen uptakes at the relative pressure $\left(P / P_{0}\right)$ range from 0.2 to 0.9 , demonstrating generation of well-defined porous framework or interparticle void spaces during thermal carbonization of the ZIF-GO composite. ${ }^{36}$ The appearance of small hysteresis loop in nanohybrid could be indicative to presence of generous, random, uneven, and nonuniform mesopores as developed during thermal carbonization at high temperature, according to Yamauchi et al. ${ }^{37}$ The Brunauer-Emmett-Teller (BET) specific surface areas of the three materials $\mathrm{Co}_{3} \mathrm{O}_{4} @$ GNC-A, $\mathrm{Co}_{3} \mathrm{O}_{4} @$ GNC-B, and $\mathrm{Co}_{3} \mathrm{O}_{4} @$ GNC-C were evaluated to be 112, 187 , and $163 \mathrm{~m}^{2} \mathrm{~g}^{-1}$, respectively. However, a very small specific surface area has been obtained for $\mathrm{Co}_{3} \mathrm{O}_{4} @$ GNC-A, which could be ascribed to the formation of dense graphitic carbon nitride or the restacking of GO layers during thermal treatment at $600^{\circ} \mathrm{C}$. ${ }^{9}$ The pore volumes of $\mathrm{Co}_{3} \mathrm{O}_{4} @$ GNC-A, $\mathrm{Co}_{3} \mathrm{O}_{4} @$ GNC-B, and $\mathrm{Co}_{3} \mathrm{O}_{4} @$ GNC-C were measured to be $0.130,0.202$, and $0.165 \mathrm{~cm}^{3} \mathrm{~g}^{-1}$, respectively. Subsequent removal of dense Co-NPs serves as hard templates to generate additional pores and formation of nitrogen-doped carbon at high temperature is thought to be responsible for the comparatively large specific surface area and pore volume for $\mathrm{Co}_{3} \mathrm{O}_{4} @$ GNC-B. The surface area of $\mathrm{Co}_{3} \mathrm{O}_{4} @$ GNC-C is found to be low in comparison with $\mathrm{Co}_{3} \mathrm{O}_{4} @$ GNC-B due to collapse of some well-defined nanopores caused by the graphitization of amorphous carbon during the pyrolysis of nanocomposite at the higher temperature treatment process. ${ }^{38}$ The corresponding pore size distributions as calculated from 
the adsorption branches of isotherms based on the BJH (Barrett-Joyner-Halenda) method (Fig. 1c) reveal the narrow pore size distribution with the micropores predominately centered at $\sim 1.17 \mathrm{~nm}$ range. The micropores in these nitrogen doped carbonaceous materials have been developed due to the thermal decomposition of the organic framework unit followed by evaporation in the form of small molecules like $\mathrm{H}_{2} \mathrm{O}, \mathrm{CO}_{2}, \mathrm{NO}_{x}$ during the heat treatment under inert atmosphere, is consistent with the previous report by Yamauchi and co-workers. ${ }^{39}$

In order to gain insight about the surface electronic property, elemental compositions and oxidation state, we have performed XPS of all the $\mathrm{Co}_{3} \mathrm{O}_{4} @$ GNC nanohybrids. All the binding energies were calibrated through referencing to the $\mathrm{C} 1 \mathrm{~s}$ binding energy (284.6 eV). XPS survey spectra in the full scan range illustrated the presence of the $\mathrm{C}, \mathrm{N}, \mathrm{O}$, and Co at the binding energy 284.6, 398.3, 530.8, 780.2 and $795.9 \mathrm{eV}$, respectively (Fig. S6b, SI). The high-resolution $\mathrm{C}$ 1s XPS spectra (Fig.S7, SI) of $\mathrm{Co}_{3} \mathrm{O}_{4} @$ GNC nanohybrid exhibited three peaks at $\sim 284.4, \sim 285.2, \sim 286.6$ and $\sim 290 \mathrm{eV}$. The first two contributions are from the $\mathrm{sp}^{2}-$ hybridized graphite like carbon and the $\mathrm{sp}^{3}$-hybridized diamond-like carbon whereas the later two are related to the surface oxygen and nitrogen groups including $\mathrm{C}-\mathrm{N}, \mathrm{C}=\mathrm{N}, \mathrm{C}=\mathrm{O}, \mathrm{C}-\mathrm{O}-\mathrm{C}$, respectively. ${ }^{40,41}$ The high intensity of primary peak at $\sim 284.4 \mathrm{eV}$ refers to the presence of large amounts of $\mathrm{sp}^{2}$-hybridized carbon inside the sample. The appearance of the other associated peaks reflects to the formation of surface oxygen and nitrogen functional groups which are likely to be produced during the course of pyrolysis under high temperature. Highresolution Co 2p XPS spectra for $\mathrm{Co}_{3} \mathrm{O}_{4} @$ GNC nanohybrid is found to be similar to that of the bulk $\mathrm{Co}_{3} \mathrm{O}_{4}{ }^{42}$ but for the spectral weight of various components (See Fig. S8, SI and Fig. 2(a)). The spectral deconvolution for $\mathrm{Co}-2 \mathrm{p}_{3 / 2}$ region contains mainly five components (Fig.2b), the intense main feature containing the characteristic components $(\sim 779.5, \sim 780.6$ and $\sim 783.5 \mathrm{eV}$ ) attributed to the $\mathrm{Co}^{2+} / \mathrm{Co}^{3+}$ surface states of a typical $\mathrm{Co}_{3} \mathrm{O}_{4}$ system and two relatively weak components related to the shakeup satellite features. However, the spectral weights of these components are significantly different compared to a standard bulk $\mathrm{Co}_{3} \mathrm{O}_{4}$ system, a similar observation is seen in related nanosystems. ${ }^{43-45}$ Interestingly in our case, the shakeup satellites have larger spectral weight, which we attribute to the presence of further components coming from the interaction $\mathrm{Co}_{3} \mathrm{O}_{4}$ and nitrogen-doped carbon skeleton through Co-O-C and Co-N-C bonds. 

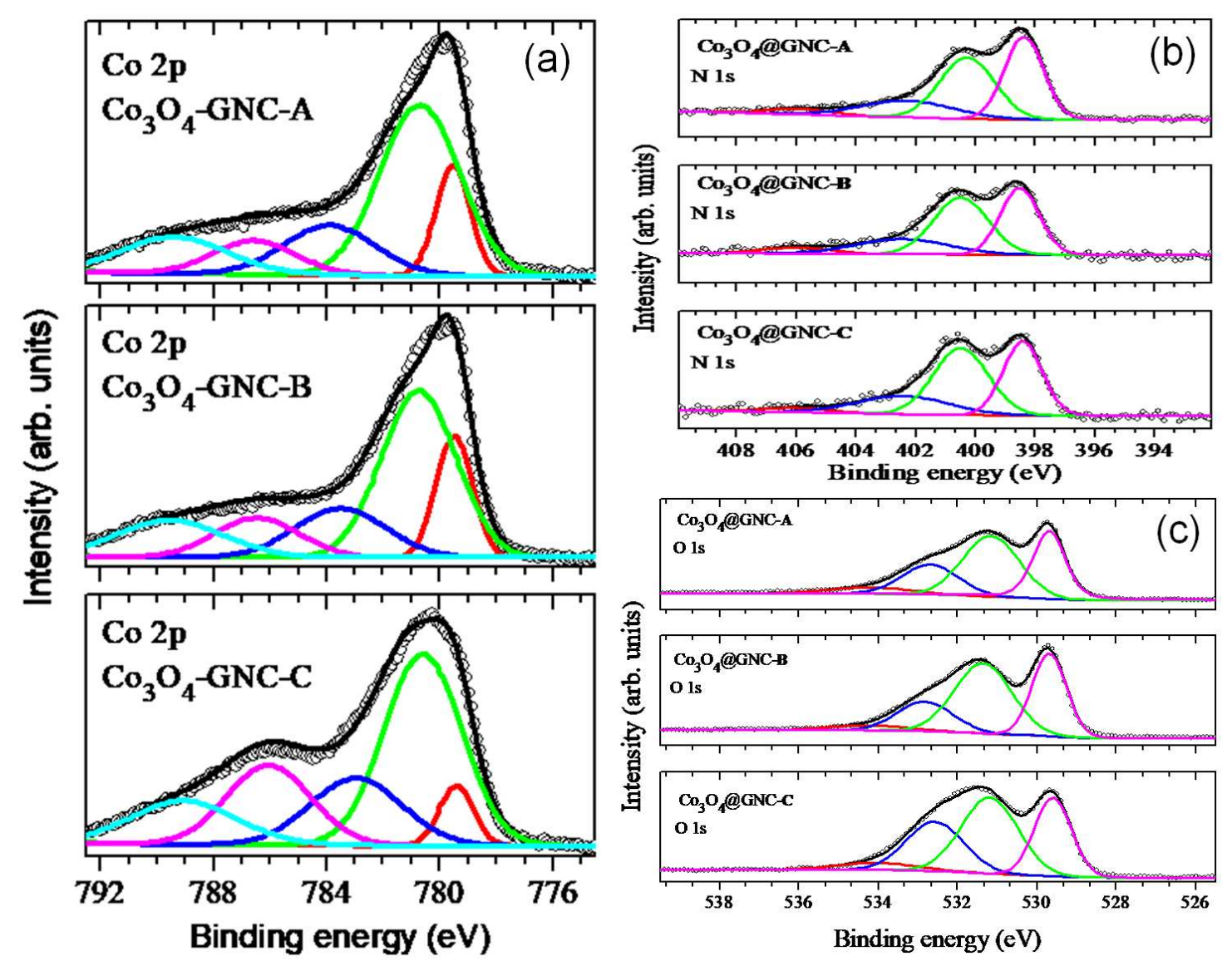

Figure 2: Deconvoluted Co-2p (a), N-1s (b), and O-1s (c) XPS spectra of $\mathrm{Co}_{3} \mathrm{O}_{4} @$ GNC-A, $\mathrm{Co}_{3} \mathrm{O}_{4} @$ GNC-B and $\mathrm{Co}_{3} \mathrm{O}_{4} @$ GNC-C, respectively.

The high resolution N-1s XPS spectra could be deconvoluted to four sub-peaks (Fig. 2(b)), where three of them having significant intensity (intensity of the fourth one is insignificant compared to the remaining three). The binding energy of the three subpeaks are at 2398.4 , $\sim 400.5$ and $\sim 402.3 \mathrm{eV}$, respectively, corresponding to the existence of characteristic pyridinic-N, graphitic-N and pyridinic $\mathrm{N}^{+}-\mathrm{O}^{-}$, species, (Fig.2a), in accordance with the previous reports for ZIF derived nitrogen doped carbon. ${ }^{37,38}$ The last peak with very poor intensity (fourth one) is mostly derived from the nitrogen-doped carbon skeleton through Co$\mathrm{N}-\mathrm{C}$ bonds. This hypothesis is supported by the $\mathrm{C} 1 \mathrm{~s}$ spectra as stated in the manuscript. In comparison to the reference XPS peak for pyridinic-N-oxide groups the binding energy displacement to higher end of range could be attributed to the influence of hybridization between the valence orbitals of $\mathrm{N}$ with the underlying substrate, which reduces core hole screening and thus raises the binding energy, in accordance with the previous report by Susi et.al ${ }^{46}$ The intensity of the binding energy peak responsible for graphitic-N species becomes gradually prominent with the enhancement of pyrolysis temperature from $600^{\circ} \mathrm{C}$ to $800^{\circ} \mathrm{C}$, 
which could be attributed to the transformation of pyridinic and pyrrolic-N to graphitic-N species thus enhancing to the fraction of nitrogen-doped carbonaceous architecture. The graphitic and pyridinic nitrogen atoms play a crucial role in strong coordination with the $\mathrm{Co}^{2+}$ ions, resulting the formation of a strong $\mathrm{Co}-\mathrm{N}_{x}$ bond which is beneficial in improving catalytic activity. Moreover, the existence of pyridinic $\mathrm{N}$ and graphitic $\mathrm{N}$ species with higher proportion, could be indicative to the successful doping of $\mathrm{N}$ atoms favourable for the formation of graphitic structure leading to high dispersion of the $\mathrm{Co}_{3} \mathrm{O}_{4} \mathrm{NPs}$ throughout carbon matrix with less aggregation. As shown from the high-resolution $\mathrm{O}$ 1s spectra of $\mathrm{Co}_{3} \mathrm{O}_{4} @$ GNC (Fig. 2c) three peaks with binding energies of $\sim 529.6, \sim 532.6$ and $\sim 534 \mathrm{eV}$ could be recognized to the characteristics lattice oxygen atoms in $\mathrm{Co}_{3} \mathrm{O}_{4}$, oxygen atoms in the surface hydroxyl groups and adsorbed water respectively. ${ }^{47}$ Interestingly there is an additional component (peak at $\sim 531.2 \mathrm{eV}$ ) with significant intensity which we ascribe to the $\mathrm{O}-\mathrm{N} / \mathrm{C}$ bonds in the hybrid system. Observation of this additional component is in agreement with the observation of additional intensity of the shakeup satellites of Co $2 p$ spectra.

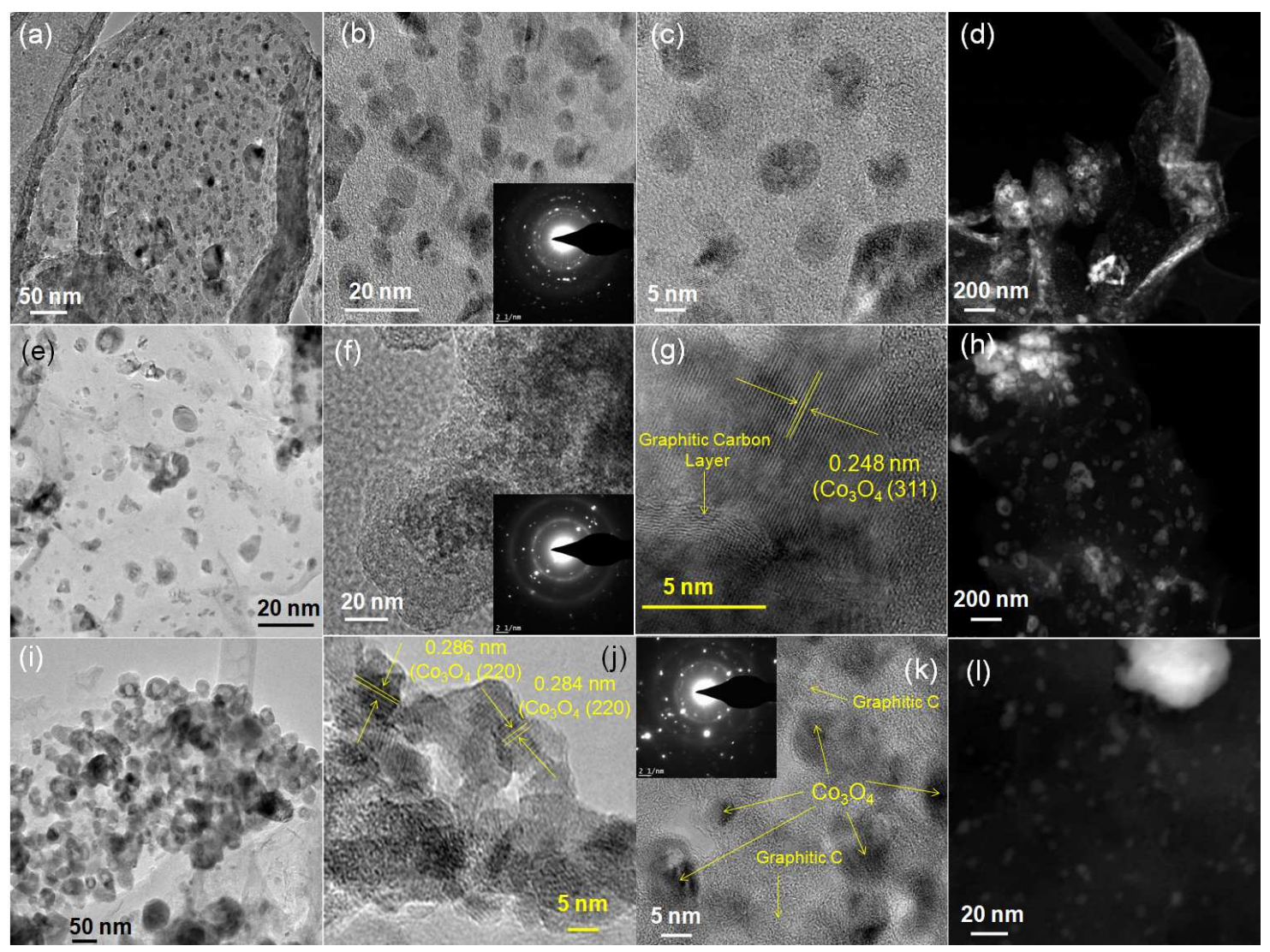

Figure 3: HR-TEM images (a, b \& c) of $\mathrm{Co}_{3} \mathrm{O}_{4} @$ GNC-A, (e, f \& g) of $\mathrm{Co}_{3} \mathrm{O}_{4} @$ GNC-B and $(\mathrm{i}, \mathrm{j} \& \mathrm{k}) \mathrm{Co}_{3} \mathrm{O}_{4} @$ GNC-C, respectively. The respective selected area electron diffraction 
patterns (SAED) are provided in the inset of the Fig.b, $\mathrm{f}$ and k. HAADF-STEM images of $\mathrm{Co}_{3} \mathrm{O}_{4} @$ GNC-A (d), $\mathrm{Co}_{3} \mathrm{O}_{4} @$ GNC-B (h) and $\mathrm{Co}_{3} \mathrm{O}_{4} @$ GNC-C (l).

The distribution, morphology of $\mathrm{Co}_{3} \mathrm{O}_{4}$-NPs and microstructures of $\mathrm{Co}_{3} \mathrm{O}_{4}$ @ GNC were thoroughly characterized by HR-TEM and HAADF-STEM tools. TEM images of the $\mathrm{Co}_{3} \mathrm{O}_{4} @$ GNC nanohybrid materials show that the black dots of spherical $\mathrm{Co}_{3} \mathrm{O}_{4}-\mathrm{NPs}$ surrounded by lighter regions of carbonaceous matrix in $\mathrm{Co}_{3} \mathrm{O}_{4} @$ GNC-A (Fig.3a \&b) and $\mathrm{Co}_{3} \mathrm{O}_{4} @$ GNC-B (Fig.3e) with a mean diameter of in the range of 7.64-11.52 and 3.77-4.96 $\mathrm{nm}$ (large and small) are uniformly distributed, which also briefly explained the promoting effect of the nitrogen content leading to smaller particle size. But in the case of $\mathrm{Co}_{3} \mathrm{O}_{4} @$ GNC-C a severe aggregation of $\mathrm{Co}_{3} \mathrm{O}_{4}-\mathrm{NPs}$ with the mean size of about 21.1-31.7 $\mathrm{nm}$ have been observed (Fig.3i). It could also be noticed from the HR-TEM images (Fig.3k) that $\mathrm{Co}_{3} \mathrm{O}_{4}$ particles (marked with arrows) are tightly wrapped by graphene-like carbon shell characteristic multiple graphitic layers. ${ }^{8}$ TEM image (Fig. S9, SI) from which the multiple graphitic layers are clearly visible resembling to the $C(002)$ plane. This nitrogen doped multiple graphitic layers as derived by carbonization of ZIF-GO composite serve as strong anchoring groups between the $\mathrm{Co}_{3} \mathrm{O}_{4}$ NPs and the carbon shells thereby inhibiting the particles from undergoing possible agglomeration and detachment. Fig.3k evidently displayed the existence of rGO in some edges of the $\mathrm{Co}_{3} \mathrm{O}_{4} @$ GNC-C material since rGO is also simultaneously formed during the course of pyrolysis of the ZIF-GO composite material. In addition, the HR-TEM images with closer inspection (Fig.3c, g \& j) further established that the crystalline $\mathrm{Co}_{3} \mathrm{O}_{4}$ NPs with spherical morphology are strongly wrapped in circular graphitic nanoporous carbon cages which formed during the catalytic graphitization. HRTEM images (Fig.3c, g \& j) revealed distinguished crystalline lattice fringes having dspacing of about 0.247 and $0.287 \mathrm{~nm}$, respectively, corresponding to the (311) and (220) crystallographic planes of the spinel $\mathrm{Co}_{3} \mathrm{O}_{4}$ nanoparticles. Distinctive bright rings associated with evident spots as noticed from the Selected Area Electron Diffraction (SAED) patterns of all $\mathrm{Co}_{3} \mathrm{O}_{4} @$ GNC nanostructures (inset of Fig.3b, f \& k) imply a polycrystalline nature of $\mathrm{Co}_{3} \mathrm{O}_{4}$. The successful decoration of $\mathrm{Co}_{3} \mathrm{O}_{4}$-NPs (bright spots) on the nitrogen doped carbon matrix was further confirmed from HAADF-STEM studies (Fig.3d, h \& 1). Particle-size distribution histogram profiles of the three catalysts is presented in the Fig. S10, SI. FE-SEM images (Fig.4) demonstrated morphological feature of $\mathrm{Co}_{3} \mathrm{O}_{4} @$ GNC-B with an obvious irregular structure, where short ribbon like particles with length of about 400-500 nm are strongly adhered together into clusters with very smooth surface. The corresponding 
elemental mapping results further confirmed the homogeneous distribution of $\mathrm{N}, \mathrm{O}$, and $\mathrm{Co}$ elements throughout the catalyst surface.

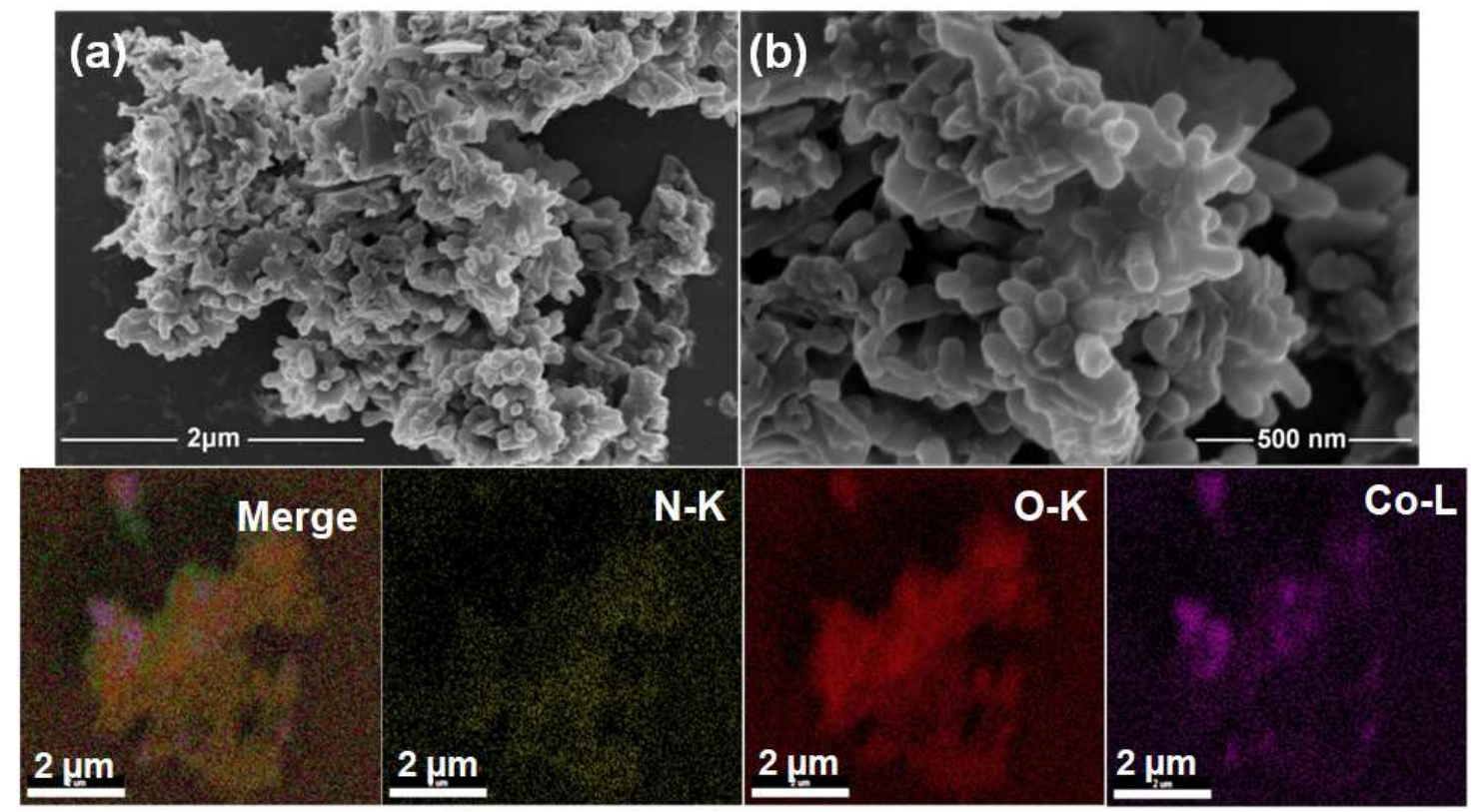

Figure 4: FE-SEM images ( $\mathrm{a} \& \mathrm{~b})$ of $\mathrm{Co}_{3} \mathrm{O}_{4} @$ GNC-B and the corresponding elemental mapping.

Fig.5 (a) presents the Co K-edge X-ray absorption near edge structure (XANES) spectra of $\mathrm{Co}_{3} \mathrm{O}_{4} @$ GNC-A, $\mathrm{Co}_{3} \mathrm{O}_{4} @$ GNC-B and $\mathrm{Co}_{3} \mathrm{O}_{4} @$ GNC-C together with that from a Co metallic foil. Compared to the metallic foil, there is a clear shift of the edge in accordance with the increase valence state of Co in the nanohybrid systems. Significant changes in the XANES features of the nanohybrid systems compared to the Co foil is in accordance to the different electronic as well as local structural arrangement in the nanohybrids. Inset in Fig. 5(a) compares the XANES of the three nanohybrids. A difference in the intensity of the feature $\sim 7730 \mathrm{eV}$ is evident, which is minimum for $\mathrm{Co}_{3} \mathrm{O}_{4} @$ GNC-B and is maximum for the $\mathrm{Co}_{3} \mathrm{O}_{4} @$ GNC-C. This may be related to the "size effect" at the nanoscale coming due to the decreasing average size of the $\mathrm{Co}_{3} \mathrm{O}_{4}$ nanoparticles in the nanohybrids. In fact a slight decrease in the oscillation amplitude of the near-edge features $(\sim 7790 \mathrm{eV})$ can be noticed for the $\mathrm{Co}_{3} \mathrm{O}_{4} @$ GNC-B where the $\mathrm{Co}_{3} \mathrm{O}_{4}$ nanoparticles average diameter are the lowest. Fourier transform magnitudes of the EXAFS oscillations extracted from the Co K-edge absorption data for the three systems are shown in Fig. 5(b)-(d). A three shell local atomic arrangement involving a set of $\mathrm{O}$, and two set of Co atoms seems to describe well the first three peaks of the Fourier transform magnitude (solid lines in Fig. 5(b)-(d)). The inverse Fourier transform of these local atomic contributions together with the corresponding EXAFS model fits are 
shown in Fig. 5(e)-(g). The obtained local atomic parameters from the EXAFS modelling are provided in Table 1. In comparison to the bulk, the EXAFS oscillations of the nanosystems show a large damping ${ }^{48-50}$ which is also true for our data. In particular, among the three nanohybrids studied, the one with larger average $\mathrm{Co}_{3} \mathrm{O}_{4}$ nanoparticle diameter shows the lowest mean square relative displacements $\left(\sigma^{2}\right)$ and the one with smaller average $\mathrm{Co}_{3} \mathrm{O}_{4}$ nanoparticle diameter shows larger $\sigma^{2}$. A small shrinkage of the local bond distances, as seen in several nanosystems are also observed for the $\mathrm{Co}_{3} \mathrm{O}_{4} @$ GNC-B in comparison to $\mathrm{Co}_{3} \mathrm{O}_{4} @$ GNC-C. We also remark that the local structural parameters extracted (Table 1) are quite similar to those obtained by Bergmann et al., investigating the catalytic activity of $\mathrm{Co}_{3} \mathrm{O}_{4}$ systems using X-ray absorption studies. ${ }^{51}$

Table 1: Local structural parameters from EXAFS modelling ( $\mathrm{N}$ stands for the coordination number and $\sigma^{2}$ stands for the means square relative displacement describing the correlated Debye Waller factor).

\begin{tabular}{|l|l|l|l|l|l|l|l|l|l|}
\hline & \multicolumn{3}{|c|}{$\mathrm{Co}_{3} \mathrm{O}_{4} @$ GNC-A } & \multicolumn{3}{c|}{$\mathrm{Co}_{3} \mathrm{O}_{4} @$ GNC-B } & \multicolumn{3}{c|}{$\mathrm{Co}_{3} \mathrm{O}_{4} @$ GNC-C } \\
\hline Shell & $\mathrm{N}$ & $\begin{array}{l}\text { Distance } \\
\AA\end{array}$ & $\begin{array}{l}\sigma^{2} \\
\AA^{2}\end{array}$ & $\mathrm{~N}$ & $\begin{array}{l}\text { Distance } \\
\AA\end{array}$ & $\begin{array}{l}\sigma^{2} \\
\AA^{2}\end{array}$ & $\mathrm{~N}$ & $\begin{array}{l}\text { Distance } \\
\AA\end{array}$ & $\begin{array}{l}\sigma^{2} \\
\AA^{2}\end{array}$ \\
\hline $\mathrm{Co}-\mathrm{O}$ & 5.5 & 1.915 & 0.007 & 5.5 & 1.913 & 0.007 & 5.5 & 1.914 & 0.006 \\
\hline $\mathrm{Co}-\mathrm{Co}$ & 4.6 & 2.858 & 0.006 & 4.6 & 2.849 & 0.006 & 4.6 & 2.853 & 0.006 \\
\hline Co-Co & 5.9 & 3.364 & 0.007 & 5.9 & 3.344 & 0.007 & 5.9 & 3.358 & 0.006 \\
\hline
\end{tabular}



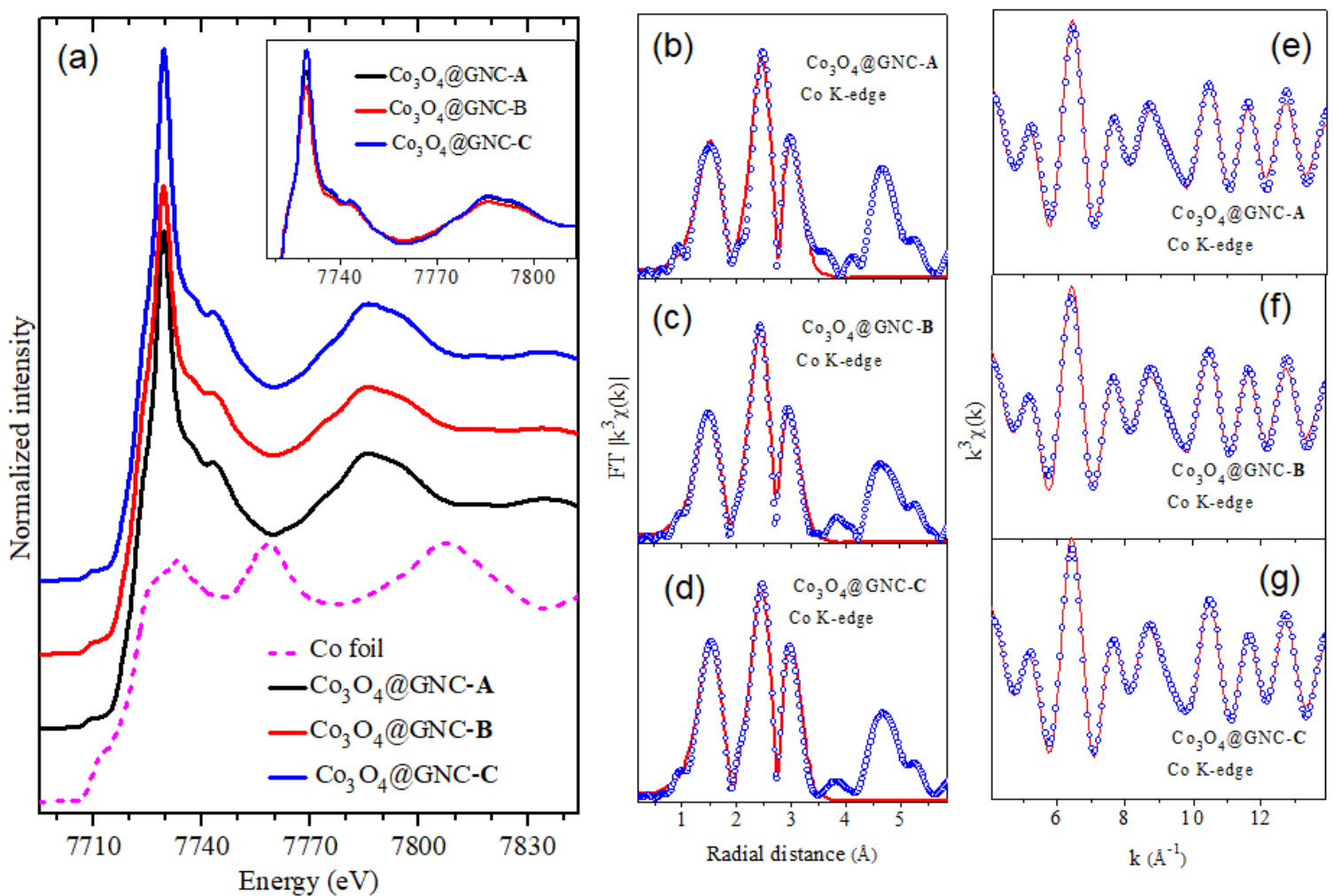

Figure 5: (a) Co K-edge XANES spectra of $\mathrm{Co}_{3} \mathrm{O}_{4} @$ GNC-A, $\mathrm{Co}_{3} \mathrm{O}_{4} @$ GNC-B and $\mathrm{Co}_{3} \mathrm{O}_{4} @$ GNC-C nanohybrids together with that of a metallic Co foil (shifted vertically). Inset here shows a zoom over the near edge features without any vertical shift. (b)-(d) Fourier transform magnitudes of extracted from the Co K-edge EXAFS oscillations for the three systems. Solid lines here are the results of the three-shell model fit. (e)-(g) Back Fourier transformed EXAFS oscillations and the 3 shell model fit results.

The catalytic activity of the newly developed $\mathrm{Co}_{3} \mathrm{O}_{4} @$ GNC materials has been evaluated by conducting selective oxidation of saturated and unsaturated hydrocarbons under solvent-free and aerobic conditions. In this investigation, our initial study began considering ethyl benzene $(25 \mathrm{~mL})$ as model substrate over $\mathrm{Co}_{3} \mathrm{O}_{4} @$ GNC catalyst in a $100 \mathrm{~mL}$ stainless steel reactor inbuilt with a pressure gauge setup under 20 bar aerobic pressure at $140^{\circ} \mathrm{C}$ temperature solvent-free conditions holding for $4 \mathrm{~h}$. The contents of products and reactants were determined using GC-FID based on authentic samples. The obtained results of catalytic reactions with the major and minor products are summarized in Table 2. Among all the examined catalysts $\mathrm{Co}_{3} \mathrm{O}_{4} @$ GNC-B exhibited an enhanced catalytic performance providing $65.8 \%$ conversion of ethyl benzene with the $72.6 \%$ selectivity of acetophenone as major product. In contrast, we have achieved $53.8 \%$ and $36.5 \%$ conversions with the corresponding selectivity of acetophenone about 68.3\% and 57.8\% of the $\mathrm{Co}_{3} \mathrm{O}_{4} @$ GNC-A and $\mathrm{Co}_{3} \mathrm{O}_{4} @$ GNC-C catalysts, respectively. Interestingly, the bare metallic $\mathrm{Co}_{3} \mathrm{O}_{4}-\mathrm{NP}$ (without 
nitrogen doping) does not show so much effective catalytic activity (Table 2, entry 4) for this selective oxidation of ethyl benzene due to the low redox properties. The synthesis of bare $\mathrm{Co}_{3} \mathrm{O}_{4}$-NPs (8.1-9.6 nm, Fig. S13, SI) has been described in the SI. We also performed the oxidation reaction over the catalyst as derived by the thermolysis of ZIF-9 only at $700^{\circ} \mathrm{C}$ (Table 2 , entry 5 ). In this case we have achieved only $28 \%$ conversion with the $65.3 \%$ acetophenone selectivity. The higher catalytic activity of the $\mathrm{Co}_{3} \mathrm{O}_{4} @$ GNC as derived by pyrolysis of ZIF-GO could be favored by the $\pi$ - $\pi$ interaction between the graphene sheets and the benzene ring of ethyl benzene and the porous nature of the nanohybrid which helps for easy diffusion of reactants molecules to interact with catalytic active centre effectively. In view of these findings, we can propose that the presence of nitrogen doped carbonaceous framework play a decisive role, thereby introducing intrinsic synergistic effect between CoN-C and carbonaceous materials, which is beneficial for the improved catalytic performance, in accordance with the previous reports. ${ }^{52}$ For comparison, $\mathrm{Co}_{3} \mathrm{O}_{4} @$ GNC-M (ex-situ synthesis of $\mathrm{Co}_{3} \mathrm{O}_{4}-\mathrm{NP}$ and nitrogen doped carbon) was tested under the same reaction conditions, and the conversion dropped dramatically to $19.7 \%$ with $45.6 \%$ selectivity of acetophenone (Table 2, entry 6). This result clearly indicated that the $\mathrm{Co}_{3} \mathrm{O}_{4} @$ GNC catalysts developed by direct carbonization technique from ZIF-GO composite with tight encapsulation and homogeneous dispersion of $\mathrm{Co}_{3} \mathrm{O}_{4}$-NPs inside the unique nitrogen-doped carbonaceous framework, thereby promoting the synergistic effect and improvement of catalytic activity. ${ }^{7}$

Table 2: Catalytic activity of different catalysts for the oxidation of ethylbenzene under solvent-free and aerobic conditions

\begin{tabular}{cccccc}
\hline Entry & Catalyst & Con (\%) & Acetophenone & $\begin{array}{c}\text { Selectivity (\%) } \\
\text { 1-Phenyl } \\
\text { Ethanol }\end{array}$ & Benzaldehyde \\
\hline $\mathbf{1}$ & $\mathrm{Co}_{3} \mathrm{O}_{4} @$ GNC-A & 53.8 & 68.3 & 27.9 & 3.8 \\
$\mathbf{2}$ & $\mathrm{Co}_{3} \mathrm{O}_{4} @ \mathrm{GNC}-\mathrm{B}$ & 65.8 & 72.6 & 22.2 & 5.2 \\
$\mathbf{3}$ & $\mathrm{Co}_{3} \mathrm{O}_{4} @ \mathrm{GNC}-\mathrm{C}$ & 36.5 & 57.7 & 37.1 & 5.2 \\
$\mathbf{4}$ & $\mathrm{Co}_{3} \mathrm{O}_{4}-\mathrm{NP}$ & 10.3 & 33.6 & 62.7 & 3.7 \\
$\mathbf{5}$ & $\mathrm{Co}_{3} \mathrm{O}_{4}-\mathrm{NC}$ & 28 & 65.3 & 29.4 & 4.4 \\
$\mathbf{6}^{\boldsymbol{a}}$ & $\begin{array}{c}\mathrm{Pyrolysis} \text { of ZIF-9) } \\
\mathrm{Co}_{3} \mathrm{O}_{4} @ \mathrm{GNC}-\mathrm{M}\end{array}$ & 19.7 & 45.6 & 50.0 & 4.4 \\
\hline
\end{tabular}




\begin{tabular}{cccccc}
\hline $\mathbf{7}$ & $\mathrm{Fe}-\mathrm{GNC}$ & 15.7 & 60.9 & 36.4 & 2.7 \\
$\mathbf{8}$ & $\mathrm{Ni-GNC}$ & 13.6 & 57.8 & 39.0 & 3.2 \\
$\mathbf{9}^{b}$ & $\mathrm{Co}_{3} \mathrm{O}_{4} @$ GNC-A & 25.6 & 54.3 & 41.0 & 4.7 \\
$\mathbf{1 0}^{b}$ & $\mathrm{Co}_{3} \mathrm{O}_{4} @$ GNC-B & 29.1 & 49.8 & 44.5 & 5.7 \\
$\mathbf{1 1}^{\boldsymbol{b}}$ & $\mathrm{Co}_{3} \mathrm{O}_{4} @$ GNC-C & 22.3 & 55.3 & 39.8 & 4.9 \\
$\mathbf{1 2}^{c}$ & $\mathrm{Co}_{3} \mathrm{O}_{4} @$ GNC-B & 5.7 & 65.3 & 30.0 & 4.7 \\
$\mathbf{1 3}$ & ZIF-9 & 10 & 56.3 & 37.5 & 5.2 \\
$\mathbf{1 4}$ & $\mathrm{Co}_{3} \mathrm{O}_{4} / \mathrm{C}$ & 25.6 & 48.9 & 46.2 & 4.9 \\
$\mathbf{1 5}$ & $\mathrm{Co}_{3} \mathrm{O}_{4} / \mathrm{N}-\mathrm{C}$ & 29.7 & 37.9 & 56.9 & 5.2 \\
$\mathbf{1 6}$ & $\mathrm{Without} \mathrm{Catalyst}$ & 5 & - & - & - \\
\hline
\end{tabular}

Reaction conditions: Ethylbenzene $(25 \mathrm{~mL})$, catalyst $(50 \mathrm{mg})$, air $(20 \mathrm{bar}), 140^{\circ} \mathrm{C}$, and $4 \mathrm{~h}$; ${ }^{a}$ Synthesis of the catalyst is provided in the SI; ${ }^{b}$ Reaction was carried out in presence of molecular $\mathrm{O}_{2} ;{ }^{c}$ Reaction was carried out in presence of hydroquinone; Conversion and selectivity were determined by GC (internal standard: 1,4-dichlorobenzene).

Fe-GNC and Ni-GNC afforded 15.7 and $13.6 \%$ conversions with acetophenone as the main product with 60.9 and $57.8 \%$ selectivity, respectively (Table 2, entries $7 \& 8$ ). We have also examined the reactivity of the $\mathrm{Co}_{3} \mathrm{O}_{4} @ \mathrm{GNC}$ catalysts in solvent-free oxidation of ethyl benzene under identical reaction conditions using $\mathrm{O}_{2}$ as an oxidant. Interestingly, diminishment in activity has been observed in this case compared with the reactions have been conducted under aerobic conditions (Table 2, entries 9, $10 \& 11$ ). This result unambiguously demonstrated that some impurities present in the air could catalyze the reaction compared with the pure $\mathrm{O}_{2}$ atmosphere, according to Silcox et al. ${ }^{53}$ In order to validate possible involvement of radical pathway we have conducted reaction in the presence of hydroquinone $(20 \mathrm{mg})$, a free radical scavenger. In this case we have attained only $5.7 \%$ conversion after $4 \mathrm{~h}$ signifying catalytic oxidation has been quenched (Table 2, entry 12). A comparison study of the turn over frequency $\left(\mathrm{h}^{-1}\right)$ along with the acetophenone productivity ( mol $_{\text {acetophenone }} \mathrm{g}_{\text {catalyst }}{ }^{-1} \mathrm{~h}^{-1}$ ) of the three new $\mathrm{Co}_{3} \mathrm{O}_{4} @$ GNC catalysts has been evaluated. Consequently, we have achieved TOFs $\left(\mathrm{h}^{-1}\right)$ of 2172 and $2215 \mathrm{~h}^{-1}$, respectively, for the $\mathrm{Co}_{3} \mathrm{O}_{4} @$ GNC-A and $\mathrm{Co}_{3} \mathrm{O}_{4} @$ GNC-C catalysts, affording acetophenone productivities of 48.3 and 65.6 mol $_{\text {acetophenone }} \mathrm{g}_{\text {catalyst }}{ }^{-1} \mathrm{~h}^{-1}$, respectively. On the contrary, the $\mathrm{Co}_{3} \mathrm{O}_{4} @ \mathrm{GNC}-\mathrm{B}$ 
catalyst exhibited an appreciably elevated activity under identical conditions, showing acetophenone productivity of about $236.7 \mathrm{~mol}_{\text {acetophenone }} \mathrm{g}_{\text {catalyst }}{ }^{-1} \mathrm{~h}^{-1}$ and TOF of $11380 \mathrm{~h}^{-1}$. We performed the catalytic reaction with the parent ZIF-9 moiety (Table 2, entry 13). An in this case we have achieved $10 \%$ conversion of ethyl benzene with $56.3 \%$ selectivity of acetophenone. From this experimental result we could conclude that carbonization process is really fruitful form the catalysis perspectives. N-doped interconnected 3D-porous network as derived from the pyrolysis of the ZIF-GO nanohybrid which facilitate the strong $\pi-\pi$ interaction between ethyl benzene and graphene sheets though adsorption on catalyst surface and the porous nature of the nanohybrid which help for easy diffusion of reactants molecules to interact with catalytic active centre effectively, thereby enhancing the catalytic performance. We have performed the catalytic auto oxidation reaction in the absence of any catalysts. In that case only 5\% conversion of ethyl benzene has been observed (Table 2, entry 16). We have achieved 25.6 and $29.7 \%$ conversion of ethyl benzene under optimized reaction conditions using $\mathrm{Co}_{3} \mathrm{O}_{4} / \mathrm{C} \& \mathrm{Co}_{3} \mathrm{O}_{4} / \mathrm{N}-\mathrm{C}$ based catalysts, respectively (Table 2 , entries 14 , 15). These experimental results clearly revealed that nitrogen doped carbonaceous framework play a critical role to synergistically promoting electrons on the $\mathrm{Co}_{3} \mathrm{O}_{4}$ surface which favours in weakening the chemisorption energy of $\mathrm{O}_{2}$ on catalyst surface, thereby favouring the catalytic oxidation reaction with facile oxygen transfer. Instead of strongly wrapping of Co oxides active sites by the nitrogen doped graphitic carbon layer (GNC) it exhibited impressive catalytic performance for the conversion of ethyl benzene compared with the conventional carbon layer encapsulated $\mathrm{Co}_{3} \mathrm{O}_{4}$-NPs (Table 2, entry 14). From this experimental result we can definitely emphasize that the porous nature of $\mathrm{Co}_{3} \mathrm{O}_{4} @ \mathrm{GNC}$ nanohybrids as experimentally examined by the $\mathrm{N}_{2}$ sorption study (Fig. 1b) play a crucial role in easy diffusion of the organic substrates through the porous channel to interact with the catalytic active centres, thereby facilitating enhancement in the catalytic activity which has been normally obstructed by the conventional carbon layers. $\mathrm{Co}_{3} \mathrm{O}_{4} / \mathrm{N}-\mathrm{C}$ acquired poor yield, suggesting involvement of important cooperative effect in $\mathrm{Co}_{3} \mathrm{O}_{4} @$ GNC catalysts where CoZIF-9, Graphene oxide are all play simultaneous key features in the fabrication of catalytic active sites. ${ }^{54}$ We have also compared the catalytic activity of the bare $\mathrm{Co}_{3} \mathrm{O}_{4}-\mathrm{NPs}$ as prepared by the conventional capping agents for the oxidation reaction (Table S3, SI).

The evolution of the catalytic performance with the progress of solvent-free oxidation of ethyl benzene against time has been investigated over $\mathrm{Co}_{3} \mathrm{O}_{4} @$ GNC catalysts under aerobic conditions at $140^{\circ} \mathrm{C}$ (Fig. 6). At initial $1 \mathrm{~h}, 1$-phenyl ethanol with $30.2 \%$ selectivity and acetophenone $(\sim 65.3 \%)$ is appeared with $42.6 \%$ conversion of ethyl benzene. This 
observation clearly confirmed that $\alpha$-hydrogen atom of ethyl benzene is abstracted in the first step to produce 1-phenyl ethanol as the primary product. In the second step, it is understood that the cobalt catalyst in presence of oxidising agent is favourable for the activation of the $\mathrm{OH}$ bond, leading to the formation of acetophenone as the major product. Only a trace amount of benzaldehyde ( $\sim 5 \%)$ was detected in the chromatograms, demonstrating that to the $\beta-\mathrm{C}-\mathrm{C}$ cleavage of 1-phenylethyloxy radical which is produced in the reaction medium took place to furnish the corresponding benzaldehyde. Similar scenario has been observed by Liu et al. in their through mechanistic investigation for solvent-free oxidation of ethyl benzene with Co@GCNs catalyst. ${ }^{7}$ Further continuation of the catalytic reaction after $3 \mathrm{~h}$ leads to the $65.8 \%$ conversion of ethyl benzene for $\mathrm{Co}_{3} \mathrm{O}_{4} @$ GNC-B catalyst but no further improvement has been achieved beyond $65 \%$ after $4 \mathrm{~h}$ along with the production acetophenone $(72.6 \%)$ and 1-phenyl ethanol (22.2\%), respectively. The similar trend is also observed for the $\mathrm{Co}_{3} \mathrm{O}_{4} @$ GNC-A catalyst under the identical reaction conditions. However, when using $\mathrm{Co}_{3} \mathrm{O}_{4} @$ GNC-C as catalyst, 36.5\% conversion of ethyl benzene was achieved after reaction for $4 \mathrm{~h}$ (Fig.6c). Also, a 57.8\% selectivity of acetophenone along with 1-phenyl ethanol $(\sim 37.6 \%)$ at the maximum conversion of ethyl benzene was realized after $6 \mathrm{~h}$ (Fig.6c). Undesired side products including acid and ester were not observed by GC-MS (detection threshold 0.3\%), signifying that $\mathrm{Co}_{3} \mathrm{O}_{4} @$ GNC is considered as an authentic selective oxidation catalyst for the aromatic hydrocarbons. ${ }^{55}$ The origin of superior catalytic efficiency of our newly designed material could be explained with the combination of the above characterization details. Higher surface area with large pore volume of $\mathrm{Co}_{3} \mathrm{O}_{4} @ \mathrm{GNC}-\mathbf{B}$ catalyst compared with the other catalysts facilitated easy diffusion of reactants into the porous channel thereby providing better interaction between catalytic active centres and substrate is thought to be responsible for the difference in catalytic activity. 

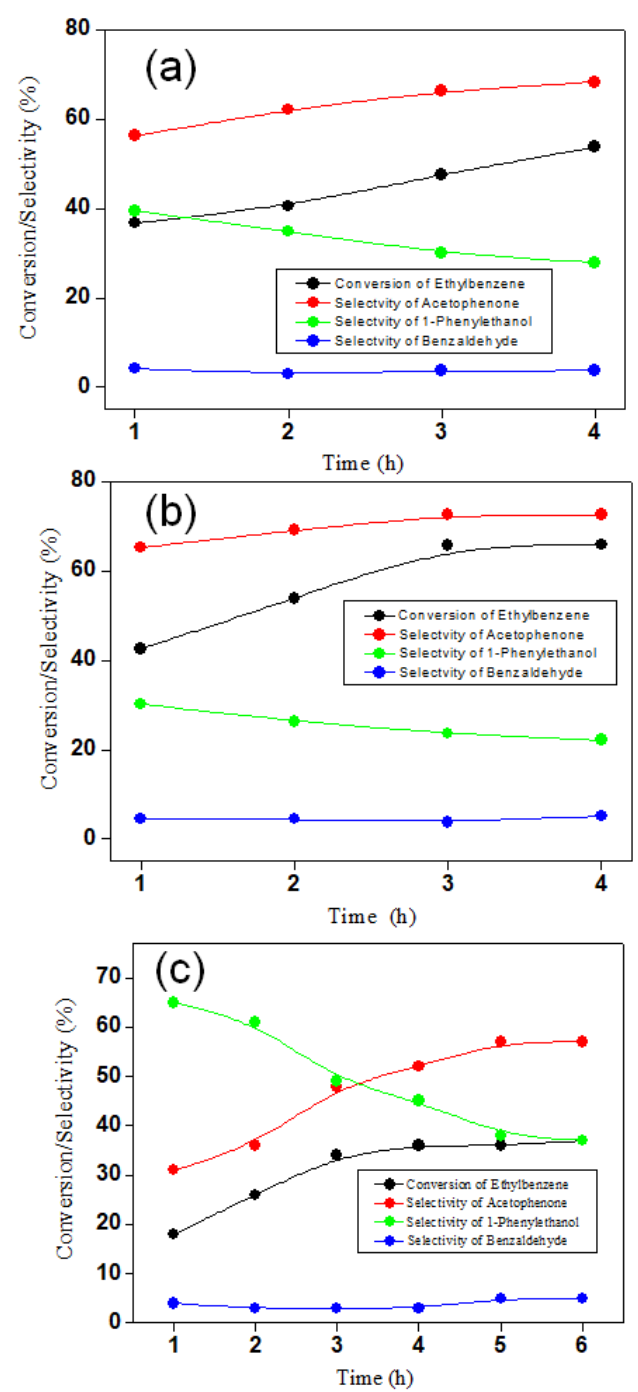

Figure 6: Evolution of reactant and product distributions as a function of time over (a) $\mathrm{Co}_{3} \mathrm{O}_{4} @$ GNC-A, (b) $\mathrm{Co}_{3} \mathrm{O}_{4} @$ GNC-B, and (c) $\mathrm{Co}_{3} \mathrm{O}_{4} @$ GNC-C catalysts for solvent-free aerobic oxidation of ethyl benzene. Reaction conditions: Ethyl benzene $(20 \mathrm{~mL})$, catalyst (60 $\mathrm{mg})$, air (20 bar), $140^{\circ} \mathrm{C}$.

In this work, the catalytic activity is gradually increased with the decrease of average $\mathrm{Co}_{3} \mathrm{O}_{4}$ NPs size from 7.6 to $3.8 \mathrm{~nm}$, but activity significantly decreased when the particle size becomes larger than $21 \mathrm{~nm}$. From this observation we can conclude that the size of $\mathrm{Co}_{3} \mathrm{O}_{4}$ play an important role in controlling the catalytic activity, which may be likely due to existence of high density of surface- sites on the nanoparticles having size about of $3.8 \mathrm{~nm}$. Similar kind of size-dependent catalytic activity on Au clusters for aerobic oxidation of cyclohexane was reported by Tsukuda et al and they have postulated that the decreased adsorption energy of oxygen atoms on surface of Au nanoparticles with a large size is main factor for the diminishment in activity. ${ }^{56}$ Although we provide important results on the size 
dependence, further future investigations specifically addressing this issue are required for the fundamental insight on the size-dependence catalytic activity. The improved catalytic performance of $\mathrm{Co}_{3} \mathrm{O}_{4} @$ GNC-B catalyst could also be attributed to the possible cooperative and/or synergistic effects between the $\mathrm{Co}_{3} \mathrm{O}_{4}$ nanocrystal and nitrogen doped graphitic carbon framework, as experimentally evidenced by the positive binding energy displacement in N-1s XPS spectra (Fig.3d). The considerable decrease in the N-content as supported by the C, H, N analysis (Table S1, SI) for $\mathrm{Co}_{3} \mathrm{O}_{4} @$ GNC-C catalyst during pyrolysis gave an apparent decline in intrinsic synergistic effect, catalyst durability (leaching of Co-particles is noticed in reaction medium) and increase in particle size, thus reconfirming that nitrogen doped carbonaceous framework with uniform $\mathrm{Co}_{3} \mathrm{O}_{4}-\mathrm{NPs}$ size are necessary to give a big boost to the performance of catalysts. From the deconvoluted Co $2 p_{3 / 2}$ spectra (Fig. 2) we have calculated relative amount of the $\mathrm{Co}^{3+} / \mathrm{Co}^{2+}$ order is as follow $\mathrm{Co}_{3} \mathrm{O}_{4} @$ GNC-B > $\mathrm{Co}_{3} \mathrm{O}_{4} @$ GNC-C $>\mathrm{Co}_{3} \mathrm{O}_{4} @$ GNC-A, and similar order is observed in lower binding energy shifting of Co- $2 \mathrm{p}_{3 / 2}$ and Co-2 $\mathrm{p}_{1 / 2}$, which may facilitate the high electrostatic interaction cobalt grafted on high surface area GNC with substrate feasibly on mild conditions. In the Fig. 2(c) curve fitted into three components, and the main peak at low binding energy $(\sim 529.4 \mathrm{eV})$ corresponding to lattice oxygen. In fact, surrounding chemical environment of oxide ions could significantly make some impact on the charge distribution, which definitely results binding energy peak displacement to either side depending upon the nature of the support. The shift gives an impression that the $\mathrm{Co}^{3+} / \mathrm{Co}^{2+}$ is combined with graphitic carbon nitride to form a Co-O-GNC type bond. We have noticed a chemical shift of $\sim-1.0 \mathrm{eV}$ in the XPS data which could be recognized to the electron density transfer toward oxide ion owing to the 'basic' nature of the graphitic carbon nitride. As a result, Co-O electrostatic attraction gets reduced and weakly bound oxygen $(\sim 531.6 \mathrm{eV})$ may further contribute to the $\mathrm{C}-\mathrm{H}$ bond activation. Surface area of catalyst, particle size and crystalline size of cobalt nano particles are also parallel to above order of catalysts. These are the physicochemical properties that promote the $\mathrm{Co}_{3} \mathrm{O}_{4} @$ GNC-B to show higher catalytic activity compare to the $\mathrm{Co}_{3} \mathrm{O}_{4} @$ GNC$\mathbf{A}$ and $\mathrm{Co}_{3} \mathrm{O}_{4} @$ GNC-C. A small shrinkage of the local bond distances (XAFS analysis Fig. 5), observed for the $\mathrm{Co}_{3} \mathrm{O}_{4} @$ GNC-B in comparison to $\mathrm{Co}_{3} \mathrm{O}_{4} @$ GNC-A and $\mathrm{Co}_{3} \mathrm{O}_{4} @$ GNC-C also goes in line with this.

The effect of temperature on the catalytic activity of $\mathrm{Co}_{3} \mathrm{O}_{4} @$ GNC-A, $\mathrm{Co}_{3} \mathrm{O}_{4} @$ GNC-B and $\mathrm{Co}_{3} \mathrm{O}_{4} @$ GNC-C for the aerobic oxidation reaction was evaluated at 20 bar for $4 \mathrm{~h}$ (Fig. 7). Thus, increasing the temperature from 100 to $140^{\circ} \mathrm{C}$ resulted in an enhancement in the ethyl 
benzene conversion (from 5 to $62 \%$ ), acetophenone (from 17 to $60 \%$ ) and benzaldehyde (from 5\% to 20\%). In the case of $\mathrm{Co}_{3} \mathrm{O}_{4} @$ GNC-B, a considerable improvement in ethyl benzene conversion compared with the other catalysts $\left(\mathrm{Co}_{3} \mathrm{O}_{4} @\right.$ GNC-A and $\mathrm{Co}_{3} \mathrm{O}_{4} @$ GNCC) is noticed which could be attributed to the presence of more amount of active Co metal oxide phase at this particular temperature. Oxidation process can be initiated in the presence of $\mathrm{O}_{2}$ as di-radical at higher temperatures and pressures. ${ }^{2}$ We found that such processes become significant at $140^{\circ} \mathrm{C}$ due to the potential role of di-oxygen radical as air contains $20.9 \%$ of $\mathrm{O}_{2}$ and directly influence the conversion and product selectivity (Fig. 7). Raising the temperature $\left(160^{\circ} \mathrm{C}\right)$ favours for the production of higher amount of oxygen radicals which caused considerable enhancement in conversion but the reduces the selectivity of acetophenone due to over oxidation. ${ }^{57}$ Nevertheless, with further increase of reaction time at $160^{\circ} \mathrm{C}$, the ethyl benzene conversion becomes constatnt, but significantly changes in selectivity of acetophenone owing to the over oxidation of acetophenone.
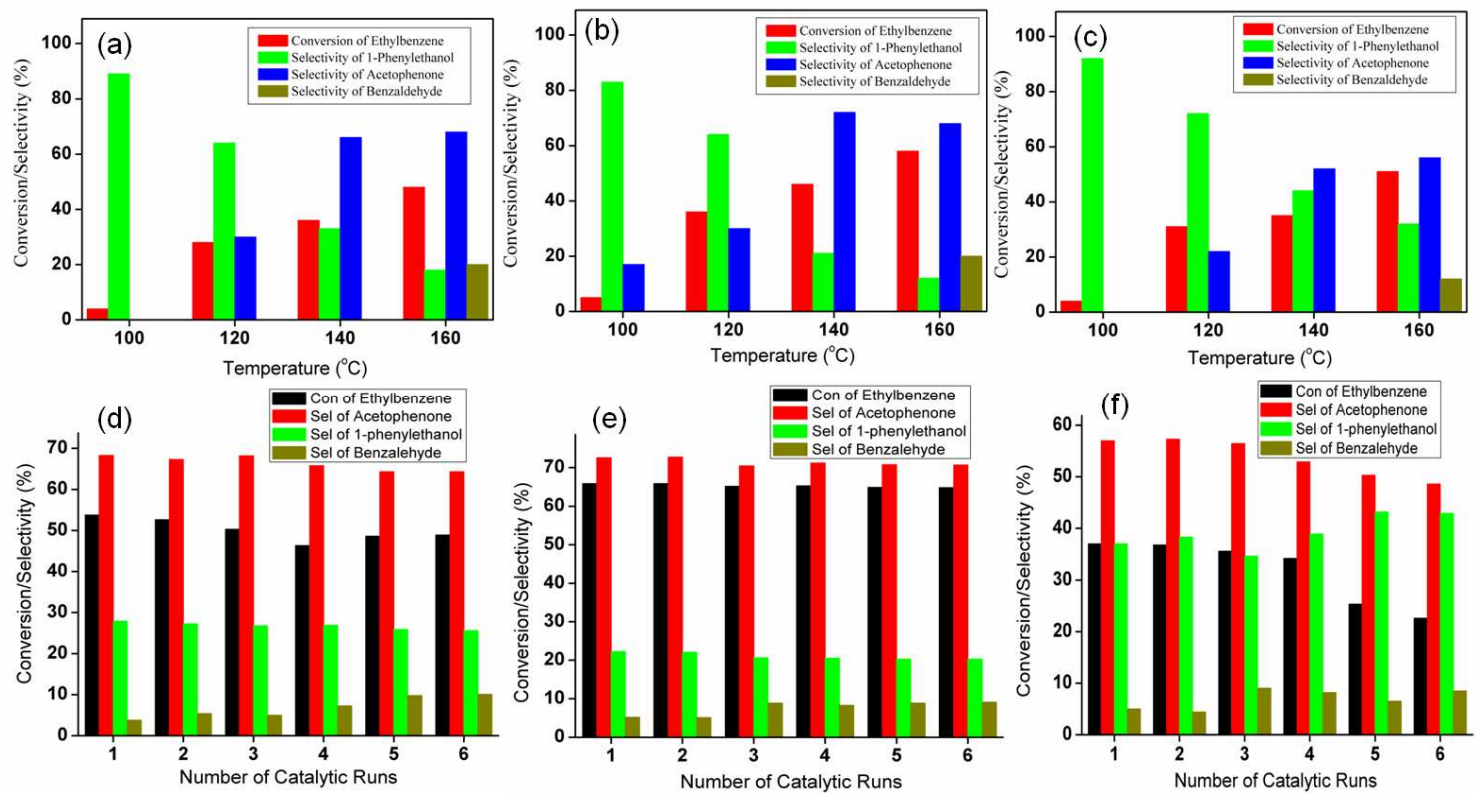

Figure 7: Influence of reaction temperature for solvent-free aerobic catalytic oxidation of ethyl benzene with (a) $\mathrm{Co}_{3} \mathrm{O}_{4} @$ GNC-A, (b) $\mathrm{Co}_{3} \mathrm{O}_{4} @$ GNC-B, and (c) $\mathrm{Co}_{3} \mathrm{O}_{4} @$ GNC-C catalysts. Recycling potential diagrams for solvent-free aerobic catalytic oxidation of ethyl benzene with (d) $\mathrm{Co}_{3} \mathrm{O}_{4} @$ GNC-A, (e) $\mathrm{Co}_{3} \mathrm{O}_{4} @$ GNC-B, and (f) $\mathrm{Co}_{3} \mathrm{O}_{4} @$ GNC-C catalysts. Reaction conditions: Ethyl benzene $(75 \mathrm{~mL})$, catalyst $(150 \mathrm{mg})$, air $(20 \mathrm{bar}), 140^{\circ} \mathrm{C}, 4 \mathrm{~h}$.

In addition, we investigated the scope of the $\mathrm{Co}_{3} \mathrm{O}_{4} @$ GNC-B catalyst for the oxidation of a variety of saturated alkanes under similar reaction conditions. Numerous secondary $\mathrm{C}-\mathrm{H}$ bonds were oxidized efficiently with excellent conversion and selectivity's to their 
corresponding oxidation products at moderate conversions (Table 3, entries 1-5). Under the optimized conditions, $\mathrm{Co}_{3} \mathrm{O}_{4} @$ GNC-B gave low conversion of toluene oxidation because of less contact time between the support and substrate (Table 3, entry 5). This could be due to the weak adsorption of toluene on the catalyst. ${ }^{58}$ Regardless of electron rich or lean group present on the saturated hydrocarbons are affecting the rate of reaction. The saturated hydrocarbons bearing electron lean substituent (Diphenylmethane, Table 3, entry 1) was oxidized very fast with high conversion (56\%) rate in comparison with electron rich groups (propylbenzene Table 3, entry 3). Comparison of conversion rate for the tetralin and indane, among these indane has shown high catalytic oxidation to respected oxidized products due to $\mathrm{C}-\mathrm{H}$ low bond dissociation energy $(82.9 \mathrm{~K} \mathrm{cal} /$ mole) and high strained $\mathrm{C}-\mathrm{H}$ bond compared to the tetralin molecule, further confirming the broader applicability of our catalysts for the selective oxidation of saturated $\mathrm{C}-\mathrm{H}$ bonds.

Table 3: Aerobic oxidation of arylalkanes, under solvent-free reaction condition, catalyzed by $\mathrm{Co}_{3} \mathrm{O}_{4} @$ GNC-B

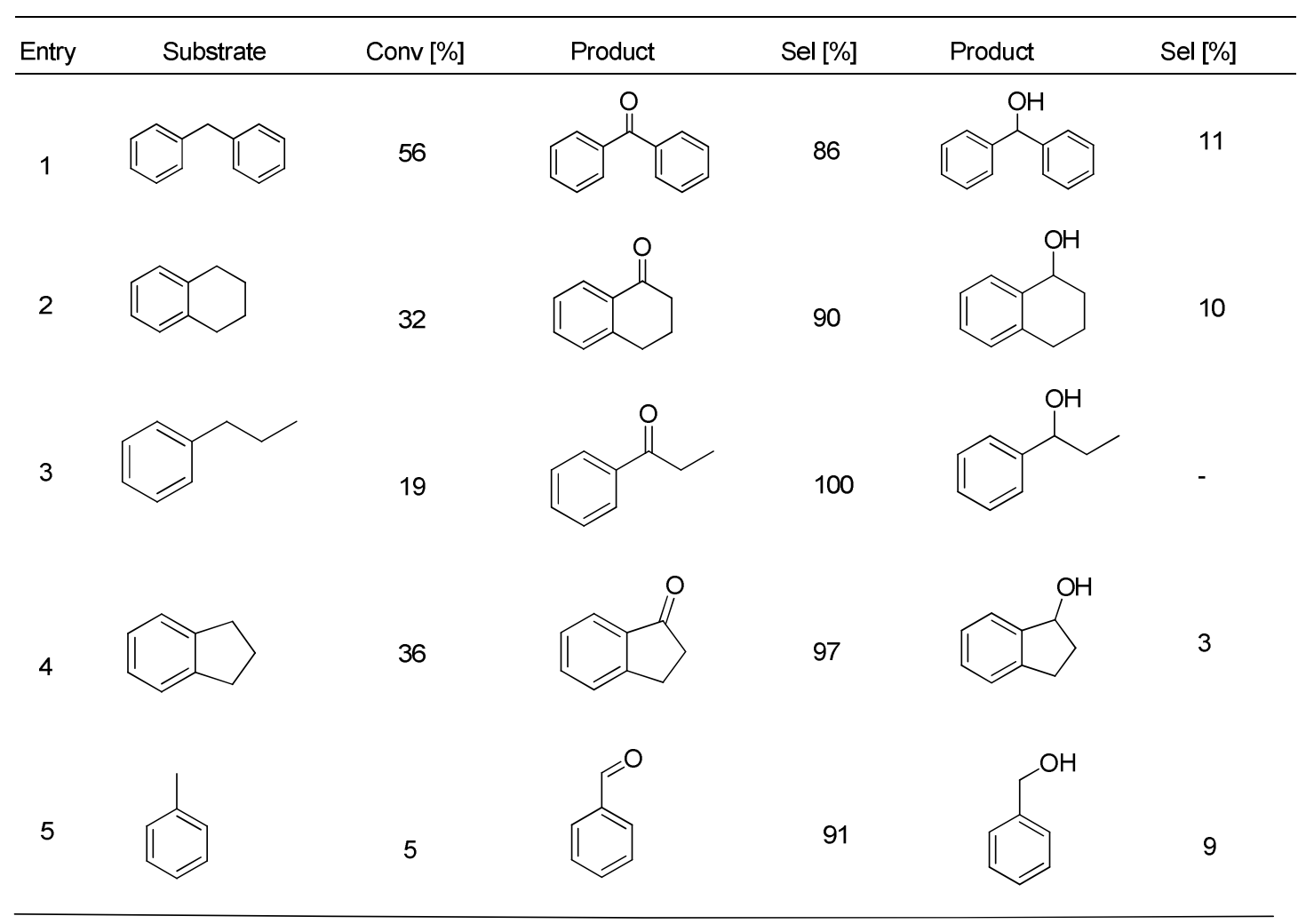

Reaction conditions: Aromatic alkanes $(20 \mathrm{~mL})$, catalyst $(60 \mathrm{mg})$, air (20 bar), $140^{\circ} \mathrm{C}$, Time (4 h). 
In our present study, our main objective is to prepare novel $\mathrm{Co}_{3} \mathrm{O}_{4}$ nanoparticles encapsulated into nitrogen-doped graphitic carbon where graphene oxide wrapped ZIF-9 microcrystals could be considered as useful precursors for the preparation of N-doped interconnected 3Dporous network and there in depth investigation on the size dependent catalytic performance of ethyl benzene oxidation to the corresponding oxidative products. From HR-TEM studies it

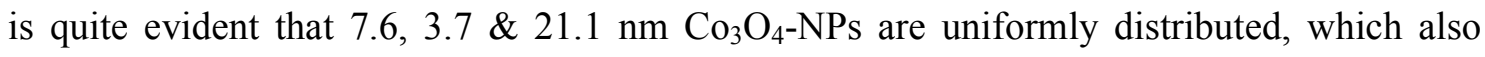
briefly explained the promoting effect of the nitrogen content in the GNC nanostructure leading to smaller particle size (Fig. 3). The considerable decrease in the N-content as supported by the $\mathrm{C}, \mathrm{H}, \mathrm{N}$ analysis (Table $\mathrm{S} 1, \mathrm{SI}$ ) for $\mathrm{Co}_{3} \mathrm{O}_{4} @$ GNC-C catalyst during pyrolysis gave an apparent decline in intrinsic synergistic effect, catalyst durability (leaching of Co-particles is noticed in reaction medium) and increase in particle size, thus reconfirming that nitrogen doped carbonaceous nanostructure with uniform $\mathrm{Co}_{3} \mathrm{O}_{4}$-NPs size are necessary to give a big boost to the performance of catalysts. Table 2 showed that the catalytic activity decreases with the increase of particle sizes. Catalytic activity of the catalyst as derived from pyrolysis of only ZIF-9 has been proved inferior compared with the catalyst as-synthesized from ZIF-GO nanohybrid, which definitely demonstrates the crucial role of the nanostructure. In view of these findings, we can also propose that the presence of nitrogen doped carbonaceous framework play a decisive role, thereby introducing intrinsic synergistic effect between Co-N-C and carbonaceous materials, which is beneficial for the improved catalytic performance, in accordance with our results for bare $\mathrm{Co}_{3} \mathrm{O}_{4}$-NPs (Table 2, entry 4). For comparison, $\mathrm{Co}_{3} \mathrm{O}_{4} @$ GNC-M (ex-situ synthesis of $\mathrm{Co}_{3} \mathrm{O}_{4}-\mathrm{NP}$ and nitrogen doped carbon) was tested under the same reaction conditions (Table 2, entry 6), and the ethyl benzene conversion dropped dramatically which also revealed the decisive function of GNC nanostructure to promote synergistic effect between $\mathrm{Co}_{3} \mathrm{O}_{4}$ nanocrystals and unique $\mathrm{N}$ containing interconnected carbonaceous framework. In order to examine the recyclability and durability of the $\mathrm{Co}_{3} \mathrm{O}_{4} @$ GNC catalysts we have conducted solvent-free aerobic oxidation reaction under optimized conditions (Fig. 7d-f) over 6 catalytic reaction cycles. After completion of each catalytic run we have recovered the solid catalyst by centrifugation technique, washed with acetone, methanol followed by a final washing in water to remove the unreacted substrate, which then dried at $100^{\circ} \mathrm{C}$ for $6 \mathrm{~h}$ and then reused in the next catalytic run. As presented in the respective Fig. $7 d \&$ e, the solvent-free ethyl benzene oxidation over $\mathrm{Co}_{3} \mathrm{O}_{4} @$ GNC-A and $\mathrm{Co}_{3} \mathrm{O}_{4} @$ GNC-B catalysts could be nicely preserved for up to six cycles of the reusability test. The ethyl benzene conversion was dropped from 53.8 to $48.9 \%$ with the diminishment of the corresponding desired product acetophenone selectivity 68.3 to 
64.3\% for $\mathrm{Co}_{3} \mathrm{O}_{4} @$ GNC-A catalyst in the sixth run, while decreasing only slightly from 65.9 to $64.8 \%$ with acetophenone selectivity 72.6 to $70.7 \%$ for $\mathrm{Co}_{3} \mathrm{O}_{4} @$ GNC-B catalyst in the subsequent cycles. This observation clearly signifies that the catalytic ethyl benzene oxidation activity was maintained with the consequent catalytic runs, but benzaldehyde selectivity has been increased. The recycling test for $\mathrm{Co}_{3} \mathrm{O}_{4} @$ GNC-A and $\mathrm{Co}_{3} \mathrm{O}_{4} @$ GNC-B catalysts after $6^{\text {th }}$ run presented a slight drop in the ethyl benzene conversion and desired product selectivity which could be attributed to the partially plug the pore entrance and gradual catalyst deactivation due to the entrapment of residual reactants and accumulation of carbonaceous deposits on the surface, as experimentally evidenced by the $\mathrm{N}_{2}$ adsorption/desorption isotherms (Fig. S11, SI) with their corresponding pore size distributions (Fig. S12, SI) of the spent $\mathrm{Co}_{3} \mathrm{O}_{4} @$ GNC catalysts. In sharp contrast, a considerable decrease in the catalytic activity of $\mathrm{Co}_{3} \mathrm{O}_{4} @$ GNC-C catalyst has been found out on multiple reuses up to $6^{\text {th }}$ catalytic runs, providing a drop from 36.5 to $22.6 \%$ in ethyl benzene oxidation (Fig. 7f). The above investigation revealed that $\mathrm{Co}_{3} \mathrm{O}_{4} @$ GNC-B is a highly stable catalyst compared with the others, affording a near-quantitative yield of acetophenone. To have an insight about this activity difference we have analysed the all the spent catalysts after sixth catalytic run by employing HAADF-STEM and TEM analysis (Fig. 8). The HAADF-STEM analysis of the $\mathrm{Co}_{3} \mathrm{O}_{4} @$ GNC catalysts recycled six times clearly exhibited distinct microstructural changes with the population of $\mathrm{Co}_{3} \mathrm{O}_{4}-\mathrm{NPs}$ that can nicely explain with the drop in catalytic activity. A closer inspection of the HAADF-STEM images showed an evidence of obvious change in the microstructure with the increase in the mean particle sizes from 7.6 to $26.9 \mathrm{~nm}$ and 3.8 to $28.9 \mathrm{~nm}$, for $\mathrm{Co}_{3} \mathrm{O}_{4} @$ GNC-A and $\mathrm{Co}_{3} \mathrm{O}_{4} @$ GNC-B, respectively. In these cases we have observed a clear homogenous distribution of $\mathrm{Co}_{3} \mathrm{O}_{4}$-NPs on the $\mathrm{N}$-doped carbonaceous matrix avoiding some local accumulation. In stark contrast, a severe aggregation of the $\mathrm{Co}_{3} \mathrm{O}_{4}$ particles has been noticed (Fig. 8) and it is very hard to locate the dispersed particles in order to measure the particle size, might be the cause for the deactivation of the $\mathrm{Co}_{3} \mathrm{O}_{4} @$ GNC-C catalyst and drastic drop in conversion over the course of the recyclability test. Additionally, some degree of Coleaching is observed during the reaction for $\mathrm{Co}_{3} \mathrm{O}_{4} @$ GNC-C catalyst after $6^{\text {th }}$ catalytic run which is thought to be responsible for the diminishment in catalyst durability and activity. This leaching is probably caused owing to a decrease in the nitrogen content by the rupture of the $\mathrm{C}-\mathrm{N}$ bonds during pyrolysis at high temperature which act as proper binding sites to anchor catalytic active sites. The Co contents of the spent $\mathrm{Co}_{3} \mathrm{O}_{4} @$ GNC-A, $\mathrm{Co}_{3} \mathrm{O}_{4} @$ GNC-B 
and $\mathrm{Co}_{3} \mathrm{O}_{4} @$ GNC-C catalysts after $6^{\text {th }}$ catalytic runs were measured to be 13.0, 2.9 and 5.5 $\mathrm{mmol} / \mathrm{g}$, respectively, by employing ICP-OES analysis.

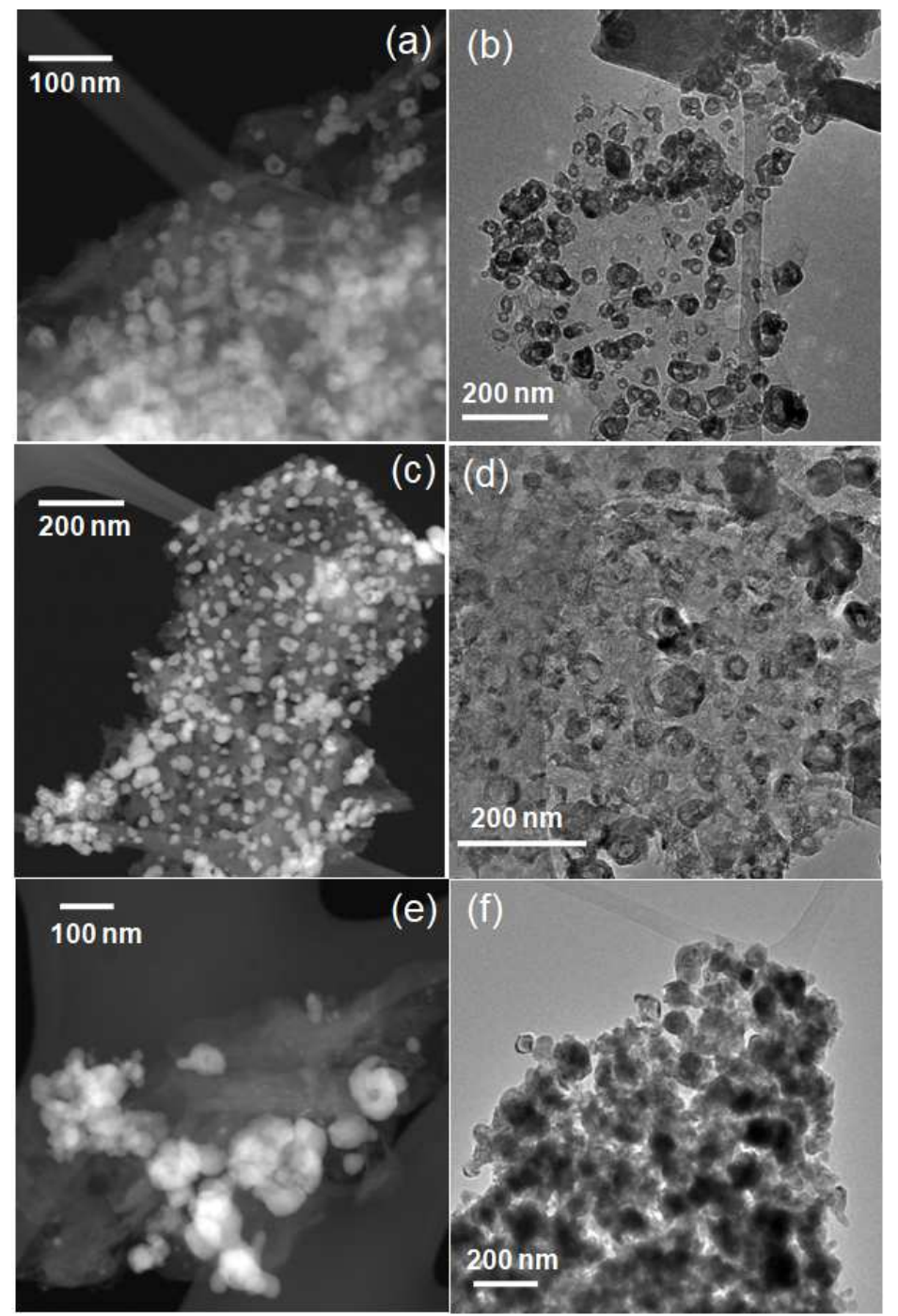

Figure 8: HAADF-STEM and TEM images of reused $\mathrm{Co}_{3} \mathrm{O}_{4} @$ GNC-A (a \& b), $\mathrm{Co}_{3} \mathrm{O}_{4} @$ GNC-B (c \& d) and $\mathrm{Co}_{3} \mathrm{O}_{4} @$ GNC-C (e \& f) after the sixth catalytic run.

We have conducted hot filtration test in order to make sure that our designed catalysts are indeed robust and heterogeneous in nature. In that case we have isolated the black color solid catalysts and achieved ethyl benzene conversion of about 52.8 and 42.8\% of $\mathrm{Co}_{3} \mathrm{O}_{4} @$ GNC-B and $\mathrm{Co}_{3} \mathrm{O}_{4} @$ GNC-A after $2 \mathrm{~h}$. We have further continued the reaction with the filtrate and observed no additional progress in ethyl benzene oxidation to desired products beyond 50 and $40 \%$, respectively. But in these cases filtrate color becomes light brown. Then we have performed AAS (atomic absorption spectroscopy) analysis of the respective filtrate and the Co content in the filtrate was below the detection limit. This limited leaching of Co in the 
reaction mixture could be explained by the inevitable intergranular friction, the collision with the stirrer and autoclave wall. Our examination undoubtedly demonstrates that our newly designed catalyst is robust and heterogeneous in nature. Indeed, the specific surface area of the reused $\mathrm{Co}_{3} \mathrm{O}_{4} @$ GNC-B catalyst after $6^{\text {th }}$ catalytic cycles reduced significantly in comparison to the fresh catalyst, which was caused possibly by the destruction of the pore wall under rapid stirring condition with the loss of porosity to hinder the accessibility of catalyst sites, during removal of entrapped solvent and unreacted substrates for further use for the next consecutive cycles (Fig. S11, SI). Decrease in the specific surface area and incredible increase in the $\mathrm{Co}_{3} \mathrm{O}_{4}$-NPs size with the severe agglomeration for $\mathrm{Co}_{3} \mathrm{O}_{4} @$ GNC-C catalyst is thought to be the main reason of catalyst deactivation and poor activity, as clearly evidenced by the combination of TEM and $\mathrm{N}_{2}$-adsoprption/desorption analysis data. We have conducted XPS analysis at Co-2p, N-1s \& O-1s core-regions of the used catalysts after the catalysis (Fig. S15, SI) which demonstrated that the chemical states of the catalytic active sites remains unaltered. We have provided the plausible mechanistic pathway for oxidation reaction catalyzed by $\mathrm{Co}_{3} \mathrm{O}_{4} @$ GNC in the respective Fig. S14, SI. The mechanism include, ethyl benzene is firstly adsorbed onto the N-doped graphitic carbon nanohybrid (GNC) surface and activated though the $\pi-\pi$ interaction between GNC and benzene ring of ethyl benzene, probably by initially weakening the $\alpha-\mathrm{C}-\mathrm{H}$ single bond of ethyl benzene. Then formation of $\mathrm{O}_{2} *$ radical form an external $\mathrm{O}_{2}$ molecule is assisted by the lattice oxygen of $\mathrm{Co}_{3} \mathrm{O}_{4}$ as well as nitrogen present in the GNC. Then weakly bonded $\alpha-\mathrm{H}$ atom of ethyl benzene is abstracted by the activated $\mathrm{O}_{2}{ }^{*}$ radical to produce the 1-phenylethyl hydro peroxide. The homolytic dissociation of 1-phenylethyl hydro peroxide molecule is significant to be the leading chain initiation step. Moreover, the large $\pi$ system of GNC and lattice oxygen of $\mathrm{Co}_{3} \mathrm{O}_{4}$ can accelerate the electron transfer-induced decomposition of 1-phenylethyl hydro peroxide, which is obvious by the evidently increased decomposition rate of benzoyl peroxide in the presence of GNC to alkoxy and hydroxy radicals. Subsequently, the formed alkoxy and hydroxyl radicals react quickly with weakly bonded $\alpha-\mathrm{H}$ atom ethyl benzene to form secondary alkyl radical. Consequently, formation of peroxy radical was occurred though attacking of oxygen with phenyl ethyl radical. After that phenyl hydrogen peroxide acts $\mathrm{H}$ atom donor for peroxy radicals to produce ketone and hydroxyl radicals. The hydroxyl radicals will react with oxygen and ethyl benzene in recycling the process to produce peroxyl radicals constantly. Secondary alkyl radicals may also react with the 1-phenylethyl hydroperoxide to generate phenyl ethyl alcohol and 1-phenylethyloxy radical, with a much 
slower rate in comparison with the benzoyl peroxide decomposition, consequential in the lower selectivity for phenyl ethyl alcohol than for ketone.

\section{CONCLUSIONS:}

In summary, we present herein a facile strategy to synthesize $\mathrm{Co}_{3} \mathrm{O}_{4}$ nanocrystals embedded into $\mathrm{N}$-doped graphitic carbon $\left(\mathrm{Co}_{3} \mathrm{O}_{4} @\right.$ GNC nanohybrids) by uniform seed-mediated growth of Co-based nanocrystals and their deposition on ZIF-9 metal-organic framework which is warped on GO nanosheets, followed by high temperature pyrolysis under inert atmosphere. We have comprehensively characterized the final $\mathrm{Co}_{3} \mathrm{O}_{4} @$ GNC nanohybrid by employing several spectroscopic tools. The $\mathrm{Co}_{3} \mathrm{O}_{4} @$ GNC composites exhibit excellent catalytic performance for selective aromatic hydrocarbon oxidations. It is found that the catalyst $\left(\mathrm{Co}_{3} \mathrm{O}_{4} @\right.$ GNC-B), as-synthesized at $700^{\circ} \mathrm{C}$ carbonization temperature holds superior catalytic activity, providing $65.8 \%$ conversion of ethyl benzene with an exclusive selectivity of $72.6 \%$ for acetophenone. Combination of XPS and XAFS spectroscopic tools surely demonstrate that the presence of $\mathrm{Co}-\mathrm{N}_{x}$ active site and the synergistic effect promote $\mathrm{Co}_{3} \mathrm{O}_{4} @ \mathrm{GNC}$ catalysts to achieve superior catalytic performance. In addition, the catalyst $\mathrm{Co}_{3} \mathrm{O}_{4} @$ GNC-B possesses better durability after recycling 6 times. High catalytic activity and good catalyst durability will attract special attention in executing further additional research on the design of promising, potential, economical, and environmentally friendly catalytic system for the selective oxidation of hydrocarbons.

Supporting Information: Characterization technique details, Elemental analysis data $(\mathrm{C}, \mathrm{H}$, N), TEM, FE-SEM images, TGA analysis of ZIF-GO composite, XPS survey spectra, C-1s XPS spectra, $\mathrm{N}_{2}$-sorption analysis of used $\mathrm{Co}_{3} \mathrm{O}_{4} @$ GNC catalysts.

\section{AUTHOR INFORMATION}

\section{Corresponding Author}

*E-mail: johncuchem@gmail.com, johnmondal@,iict.res.in

\section{Notes}

The authors declare no competing financial interest.

\section{ACKNOWLEDGMENTS}

P.S, R.S and C.S wish to thankfully acknowledge the Council of Scientific and Industrial Research (CSIR), New Delhi, for their respective junior and senior research fellowships. J.M. thanks the Department of Science and Technology, India, for DST-INSPIRE Faculty Research project grant (GAP-0522) at CSIR-IICT, Hyderabad. We are also grateful with 
Dr. Nakka Lingaiah, CSIR-IICT for his kind help in evaluation of the $\mathrm{N}_{2}$-sorption analysis data.

\section{REFERENCES:}

(1) Shilov, A. E.; Shul'pin, G. B. Activation of C-H Bonds by Metal Complexes. Chem. Rev 1997, 97, 2879-2932.

(2) Kesavan, L.; Tiruvalam, R.; Rahim, M. H. A.; Saiman, M. I. b.; Enache, D. I.; Jenkins, R. L.; Dimitratos, N.; Sanchez, J. A. L.; Taylor, S. H.; Knight, D. W.; Kiely, C. J.; Hutchings, G. J. Solvent-Free Oxidation of Primary Carbon-Hydrogen Bonds in Toluene using Au-Pd Alloy Nanoparticles. Science 2011, 331, 195-199.

(3) Zhang, J.; Liu, X.; Blume, R.; Zhang, A.; Schlögl, R.; Su, D. S. Surface-Modified Carbon Nanotubes Catalyze Oxidative Dehydrogenation of n-Butane. Science 2008, 322, 73-77.

(4) Zhang, P.; Gong, Y.; Li, H.; Chen, Z.; Wang, Y. Solvent-Free Aerobic Oxidation of Hydrocarbons and Alcohols with Pd@N-doped Carbon from Glucose. Nat. Commun. 2013, 4, 1593.

(5) Qiao, Z.; Zhang, P.; Chai, S. H.; Chi, M.; Veith, G. M.; Gallego, N. C.; Kidder, M.; Dai, S. Lab-in-a-shell: Encapsulating Metal Clusters for Size Sieving Catalysis. J. Am. Chem. Soc. 2014, 136 (32), 11260-11263.

(6) Long, J.; Liu, H.; Wu, S.; Liao, S.; Li, Y. Selective Oxidation of Saturated Hydrocarbons using Au-Pd Alloy Nanoparticles Supported on Metal-Organic Frameworks ACS Catal. 2013, 3, 647-654.

(7) Lin, X.; Nie, Z.; Zhang, L.; Mei, S.; Chen, Y.; Zhang, B.; Zhu, R.; Liu, Z. Nitrogen-Doped Carbon Nanotubes Encapsulate Cobalt Nanoparticles as Efficient Catalysts for Aerobic and Solvent-free Selective Oxidation of Hydrocarbons. Green Chem. 2017, 19, 2164-2173.

(8) Qiu, Y.; Yang, C.; Huo, J.; Liu, Z. Synthesis of Co-NC Immobilized on Carbon Nanotubes for Ethylbenzene Oxidation. J. Mol. Catal. A: Chem. 2016, 424, 276-282.

(9) Lin, X.; Zhao, S.; Chen, Y.; Fu, L.; Zhu, R.; Liu, Z. Nitrogen-Doped Carbon Cobalt Grafted on Graphitic Carbon Nitride Catalysts with Enhanced Catalytic Performance for Ethylbenzene Oxidation. J. Mol. Catal. A: Chem. 2016, 420, 11-17.

(10) Luo, J.; Yu, H.; Wang, H.; Peng, F. Enhancing the Catalytic Activity of Carbon Nanotubes by Filled Iron Nanowires for Selective Oxidation of Ethylbenzene. Catal. Commun. 2014, 51, 77-81.

(11) Liao, S.; Chi, Y.; Yu, H.; Wang, H.; Peng, F. Tuning the Selectivity in the Aerobic Oxidation of Cumene Catalyzed by Nitrogen $\square$ Doped Carbon Nanotubes. ChemCatChem 2014, 6, 555-560.

(12) Luo, J.; Peng, F.; Yu, H.; Wang, H.; Zheng, W. Aerobic Liquid $\square$ Phase Oxidation of Ethylbenzene to Acetophenone Catalyzed by Carbon Nanotubes. ChemCatChem 2013, 5, 1578-1586.

(13) Tan, B.; Wu, Z. F.; Xie, Z. L. Fine Decoration of Carbon Nanotubes with Metal Organic Frameworks for Enhanced Performance in Supercapacitance and Oxygen Reduction Reaction. Sci. Bull. 2017, 62, 1132-1141.

(14) Kreno, L. E.; Leong, K.; Farha, O. K.; Allendorf, M.; Duyne, R. P. V.; Hupp, J. T. Metal-Organic Framework Materials as Chemical Sensors. Chem. Rev. 2012, 112, 11051125 . 
(15) Sumida, K.; Rogow, D. L.; Mason, J. A.; McDonald, T. M.; Bloch, E. D.; Herm, Z. R.; Bae, T. H.; Long, J. R. Carbon Dioxide Capture in Metal-Organic Frameworks. Chem. Rev. 2012, 112, 724-781.

(16) Liu, B.; Shioyama, H.; Akita T.; Xu, Q. Metal-Organic Framework as a Template for Porous Carbon Synthesis. J. Am. Chem. Soc. 2008, 130, 5390-5391.

(17) Chaikittisilp, W.; Ariga, K.; Yamauchi, Y. A New Family of Carbon Materials: Synthesis of MOF-Derived Nanoporous Carbons and Their Promising Applications. $J$. Mater. Chem. A 2013, 1, 14-19.

(18) Liu, Y.; Huang, B.; Xie, Z. Hydrothermal Synthesis of Core-Shell $\mathrm{MoO}_{2} / \alpha-\mathrm{Mo}_{2} \mathrm{C}$ Heterojunction as High Performance Electrocatalyst for Hydrogen Evolution Reaction. Appl. Surf. Sci. 2018, 427, 693-701.

(19) Yu, G.; Sun, J.; Muhammad, F.; Wang, P.; Zhu, G. Cobalt-Based Metal Organic Framework as Precursor to Achieve Superior Catalytic Activity for Aerobic Epoxidation of Styrene. RSC Adv. 2014, 4, 38804-38811.

(20) Wei, C.; Li, X.; Xu, F.; Tan, H.; Li, Z.; Sun, L.; Song, Y. Metal Organic FrameworkDerived Anthill-like Cu@carbon Nanocomposites for Nonenzymatic Glucose Sensor. Anal. Methods 2014, 6, 1550-1557.

(21) Hu, J.; Wang, H.; Gao Q.; Guo, H. Porous Carbons Prepared by Using Metal-Organic Framework as the Precursor for Supercapacitors. Carbon 2010, 48, 3599-3606.

(22) Torad, N. L.; Hu, M.; Ishihara, S.; Sukegawa, H.; Belik, A. A.; Imura, M.; Ariga, K.; Sakka, Y.; Yamauchi, Y. Direct Synthesis of MOF $\square$ derived Nanoporous Carbon with Magnetic Co Nanoparticles toward Efficient Water Treatment. Small 2014, 10, 20962107.

(23) Zou, F.; Hu, X.; Li, Z.; Qie, L.; Hu, C.; Zeng, R.; Jiang, Y.; Huang, Y. MOF $\square$ Derived Porous $\mathrm{ZnO} / \mathrm{ZnFe}_{2} \mathrm{O}_{4} / \mathrm{C}$ Octahedra with Hollow Interiors for High $\square$ Rate Lithium $\square$ Ion Batteries. Adv. Mater. 2014, 26, 6622-6628.

(24) Lin, K. Y. A.; Hsu. F. K.; Lee, W. D. Magnetic Cobalt-Graphene Nanocomposite Derived from Self-Assembly of MOFs with Graphene Oxide as an Activator for Peroxymonosulfate. J. Mater. Chem. A 2015, 3, 9480-9490.

(25) Wei, J.; Hu, Y.; Liang, Y.; Kong, B.; Zheng, Z.; Zhang, J.; Jiang, S. P.; Zhao, Y.; Wang, H. Graphene Oxide/Core-Shell Structured Metal-Organic Framework NanoSandwiches and their Derived Cobalt/N-doped Carbon Nanosheets for Oxygen Reduction Reactions. J. Mater. Chem. A 2017, 5, 10182-10189.

(26) Wei, J.; Hu, Y.; Wu, Z.; Liang, Y.; Leong, S.; Kong, B.; Zhang, X.; Zhao, D.; Simone G. P.; Wang, H. A Graphene-Directed Assembly Route to Hierarchically Porous Co- $\mathrm{N}_{x} / \mathrm{C}$ Catalysts for High-Performance Oxygen Reduction. J. Mater. Chem. A 2015, 3, 16867-16873.

(27) Li, X.; Fang, Y.; Wen, L.; Li, F.; Yin, G.; Chen, W.; An, X.; Jin, J.; Ma, J. Co@ $\mathrm{Co}_{3}$ $\mathrm{O}_{4}$ Core-Shell Particle Encapsulated N-doped Mesoporous Carbon Cage Hybrids as Active and Durable Oxygen-Evolving Catalysts. Dalton Trans. 2016, 45, 5575-5582.

(28) Tang, L.; Chang, H.; Liu, Y.; Li, J. Duplex DNA/Graphene Oxide Biointerface: From Fundamental Understanding to Specific Enzymatic Effects. Adv. Funct. Mater. 2012, 22, 3083-3088. 
(29) Cong, J.; Lei, F.; Zhao, T.; Liu, H.; Wang, J.; Lu, M.; Li, Y.; Xu, H. ; Gao, J. Two Co-zeolite Imidazolate Frameworks with Different Topologies for Degradation of Organic Dyes via Peroxymonosulfate Activation. J Solid State Chem., 2017, 256, 10-13.

(30) Li, X.; Fang, Y.; Lin, X.; Tian, M.; An, X.; Fu, Y.; Li, R.; Jin, J.; Ma, J. MOF Derived $\mathrm{Co}_{3} \mathrm{O}_{4}$ Nanoparticles Embedded in N-Doped Mesoporous Carbon Layer/MWCNTs Hybrids: Extraordinary Bi-functional Electrocatalysts for OER and ORR. J. Mater. Chem. A, 2015, 3, 17392-17402.

(31) Liang, Y.; Li, Y.; Wang, H.; Zhou, J.; Wang, J.; Regier, T.; Dai, H. $\mathrm{Co}_{3} \mathrm{O}_{4}$ Nanocrystals on Graphene as a Synergistic Catalyst for Oxygen Reduction Reaction. Nat. Mater. 2011, 10, 780-786.

(32) Zhuang, Z.; Sheng, W.; Yan, Y. Synthesis of monodispere $\mathrm{Au} @ \mathrm{Co}_{3} \mathrm{O}_{4}$ Core $\square$ Shell Nanocrystals and their Enhanced Catalytic Activity for Oxygen Evolution Reaction. Adv. Mater. 2014, 26, 3950-3955.

(33) Liu, Y.; Jiao, L.; Wu, Q.; Zhao, Y.; Cao, K.; Liu, H.; Wang, Y.; Yuan, H. Synthesis of rGO-Supported Layered $\mathrm{MoS}_{2}$ for High-performance Rechargeable Mg Batteries. Nanoscale 2013, 5, 9562-9567.

(34) Mathew, S.; Joseph, B.; Sekhar, B. R.; Dev, B. N. X-ray Photoelectron and Raman Spectroscopic Studies of MeV Proton Irradiated Graphite. Nucl. Instrum. Meth. Phys. 2008, 226, 3241-3246.

(35) Zheng, X.; Xu, J.; Yan, K.; Wang, H.; Wang, Z.; Yang, S. Space-Confined Growth of $\mathrm{MoS}_{2}$ Nanosheets within Graphite: The Layered Hybrid of $\mathrm{MoS}_{2}$ and Graphene as an Active Catalyst for Hydrogen Evolution Reaction. Chem. Mater. 2014, 26, 2344-2353.

(36) Long, J.; Shen, K.; Chen, L.; Li, Y. Multimetal-MOF-derived Transition Metal Alloy NPs Embedded in an N-doped Carbon Matrix: Highly Active Catalysts for Hydrogenation Reactions. J. Mater. Chem. A 2016, 4, 10254-10262.

(37) Tang, J.; Salunkhe, R. R.; Liu, J.; Torad, N. L.; Imura, M.; Furukawa, S.; Yamauchi Y. Thermal Conversion of Core-Shell Metal-Organic Frameworks: A New Method for Selectively Functionalized Nanoporous Hybrid Carbon. J. Am. Chem. Soc. 2015, 137, 1572-1580.

(38) Tang, J.; Salunkhe, R. R.; Zhang, H.; Malgras V.; Ahamad, T.; Alshehri, S. M.; Kobayashi, N.; Tominaka, S.; Ide, Y.; Kim, J. H.; Yamauchi, Y. Bimetallic MetalOrganic Frameworks for Controlled Catalytic Graphitization of Nanoporous Carbons. Sci. Rep. 2016, 6, 30295.

(39) Kaneti, Y.V.; Zhang, J.; He, Y. B.; Wang, Z.; Tanaka, S.; Hossain, M. S. A.; Pan, Z.; Xiang, B.; Yang, Q.H.; Yamauchi, Y. Fabrication of An MOF-derived HeteroatomDoped $\mathrm{Co} / \mathrm{CoO} /$ carbon Hybrid with Superior Sodium Storage Performance for SodiumIon Batteries. J. Mater. Chem. A 2017, 5, 15356-15366.

(40) Zhang, L.; Su, Z.; Jiang, F.; Yang, L.; Qian, J.; Zhou, Y.; Li, W.; Hong, M. Highly Graphitized Nitrogen-doped Porous Carbon Nanopolyhedra Derived from ZIF-8 Nanocrystals as Efficient Electrocatalysts for Oxygen Reduction Reactions. Nanoscale 2014, 6, 6590-6602. 
(41) Zhang, X.; Liu, S.; Zang, Y.; Liu, R.; Liu, G.; Wang, G.; Zhang, Y.; Zhang, H.; Zhao, H. Co/Cog $\mathrm{S}_{8} @ \mathrm{~S}, \mathrm{~N}$-doped Porous Graphene Sheets Derived from S, N Dual Organic Ligands Assembled Co-MOFs as Superior Electrocatalysts for Full Water Splitting in Alkaline Media Nano Energy 2016, 30, 93-102.

(42) Biesinger, M. C.; Payne, B. P.; Grosvenor, A. P.; Laua, L. W. M.; Gerson, A. R.; Smart, R. St. C. Resolving Surface Chemical States in XPS Analysis of First Row Transition Metals, Oxides and Hydroxides: Cr, Mn, Fe, Co and Ni. Appl. Surf. Sci. 2011, 257, 2717-2730.

(43) Zhang, C.; Xiao, J.; Lv, X.; Qian, L.; Yuan, S.; Wang, S.; Lei, P. Hierarchically Porous $\mathrm{Co}_{3} \mathrm{O}_{4} / \mathrm{C}$ Nanowire Arrays Derived from a Metal-Organic Framework for High Performance Supercapacitors and the Oxygen Evolution Reaction. J. Mater. Chem. A 2016, 4, 16516-16523.

(44) Wu, Z. S.; Chen, L.; Liu, J.; Parvez, K.; Liang, H. High $\square$ Performance Electrocatalysts for Oxygen Reduction Derived from Cobalt Porphyrin $\square$ Based Conjugated Mesoporous Polymers. Adv. Mater. 2014, 26, 1450-1455.

(45) Zhang, L.; Mi, T.; Ziaee, M. A.; Liang, L.; Wang, R. J. Hollow POM@ MOF HybridDerived Porous $\mathrm{Co}_{3} \mathrm{O}_{4} / \mathrm{CoMoO}_{4}$ Nanocages for Enhanced Electrocatalytic Water Oxidation. J. Mater. Chem. A 2018, 6, 1639-1647.

(46) Susi, T.; Pichler, T.; Ayala, P. X-ray Photoelectron Spectroscopy of Graphitic Carbon Nanomaterials Doped with Heteroatoms. Beilstein J. Nanotechnol. 2015, 6, 177-192.

(47) Wang, L.; Mitoraj, D.; Turner, S.; Khavryuchenko, O. V.; Jacob, T.; Hocking, R. K.; Beranek, R. Ultrasmall $\mathrm{CoO}(\mathrm{OH})_{\mathrm{x}}$ Nanoparticles As a Highly Efficient "True" Cocatalyst in Porous Photoanodes for Water Splitting. ACS Catal. 2017, 7, 4759-4767.

(48) Marakatti, V. S.; Sarma, S. C.; Joseph, B.; Banerjee, D.; Peter S. C. Synthetically Tuned Atomic Ordering in $\mathrm{PdCu}$ Nanoparticles with Enhanced Catalytic Activity toward Solvent-Free Benzylamine Oxidation. ACS Appl. Mater. Interfaces 2017, 9 (4), 36023615.

(49) Maugeri, L.; Simonelli, L.; Iadecola, A.; Joseph, B.; Okubo, M.; Honma, I.; Wadati, H.; Mizokawa, T.; Saini, N.L. Temperature Dependent Local Structure of $\mathrm{LiCoO}_{2}$ Nanoparticles Determined by Co K-edge X-ray Absorption Fine Structure. J. Power Sources 2013, 229, 272-276.

(50) Suber, L.; Imperatori, P.; Pilloni, L.; Caschera, D.; Angelini, N.; Mezzi, A.; Kaciulis, S.; Iadecola, A.; Joseph, B.; Campi, G. Nanocluster Superstructures or Nanoparticles? The Self-Consuming Scaffold Decides. Nanoscale 2018, 10, 7472-7483.

(51) Bergmann, A.; Moreno, E. M.; Teschner, D.; Chernev, P.; Gliech, M.; Araújo, J. F. d.; Reier, T.; Dau, H.; Strasser, P. Reversible Amorphization and the Catalytically Active State of Crystalline $\mathrm{Co}_{3} \mathrm{O}_{4}$ During Oxygen Evolution. Nat. Commun. 2015, 6, 8625.

(52) Wang, H.; Chen, C.; Zhang, Y.; Peng, L.; Ma, S. In Situ Oxidation of CarbonEncapsulated Cobalt Nanocapsules Creates Highly Active Cobalt Oxide Catalysts for Hydrocarbon Combustion. Nat. Commun. 2015, 6, 7181.

(53) Preciado, I.; Young,T.; Silcox, G. Mercury Oxidation by Halogens under Air-and Oxygen-Fired Conditions. Energy Fuels 2014, 28, 1255-1261. 
(54) Wei, Z.; Wang, J.; Mao, S.; Su, D.; Jin, H.; Wang, Y.; Xu, F.; Li, H.; Wang, Y. In Situ-Generated $\mathrm{Co}^{0}-\mathrm{Co}_{3} \mathrm{O}_{4} / \mathrm{N}$-doped Carbon Nanotubes Hybrids as Efficient and Chemoselective Catalysts for Hydrogenation of Nitroarenes. ACS Catal. 2015, 5, 47834789.

(55) Arshadi, M.; Ghiaci, M.; Rahmanian, A.; Ghaziaskar, H.; Gil, A. Oxidation of Ethylbenzene to Acetophenone by a Mn Catalyst Supported on a Modified Nanosized $\mathrm{SiO}_{2} / \mathrm{Al}_{2} \mathrm{O}_{3}$ Mixed-Oxide in Supercritical Carbon Dioxide. Appl. Catal. B 2012, 119-120, 81-90.

(56) Liu, Y.; Tsunoyama, H.; Akita, T.; Xie, S.; Tsukuda, T. Aerobic Oxidation of Cyclohexane Catalyzed by Size-Controlled Au Clusters on Hydroxyapatite: Size Effect in the Sub-2 nm Regime. ACS Catal. 2011, 1, 2-6.

(57) Zhou, W.; Dai, X.; Chen, Y.; Sun, F.; He, M.; Chen, Q. Synergistic Catalytic Oxidation of Ethylbenzene to Acetophenone by Metallophthalocyanine Intercalated Layered Double Hydroxide with Oxygen. Chemistry Select. 2018, 3, 566-572.

(58) Bedia, J.; Rosas, J. M.; Mirasol, J. R. Cordero, T. Pd Supported on Mesoporous Activated Carbons with High Oxidation Resistance as Catalysts for Toluene Oxidation. Appl. Catal., B. 2010, 94, 8-18. 


\section{Graphical Abstract:}

Zeolitic Imidazolate Framework-Mediated Synthesis of $\mathrm{Co}_{3} \mathrm{O}_{4}$ Nanoparticles Encapsulated in N-doped Graphitic Carbon as an Efficient Catalyst for Selective Oxidation of Hydrocarbons

Saikiran Pendem, Ramana Singuru, Chitra Sarkar, Boby Joseph, Jyh-Fu Lee, Digambar B. Shinde, Zhiping Lai and John Mondal*

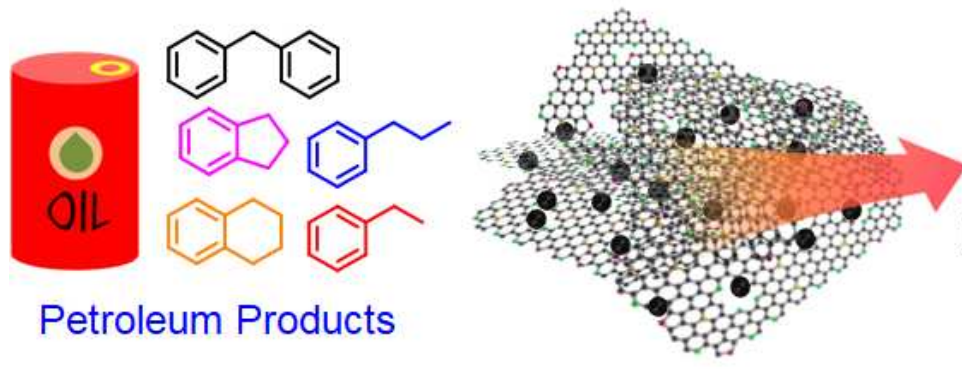<smiles>[Al][Te][Te][Te]</smiles> 